\title{
The Use of Augmented Reality in Latin-American Engineering Education: A Scoping Review
}

\author{
Roberto Santiago Bellido García ${ }^{1^{*}}$ (D), Luis Gerardo Rejas Borjas ${ }^{2}$ (D), Alejandro Cruzata-Martínez ${ }^{1}$ (D), \\ Merce Concepción Sotomayor Mancisidor ${ }^{3}$ \\ 1 Universidad San Ignacio de Loyola, PERU \\ 2 Universidad Nacional Mayor de San Marcos, PERU \\ ${ }^{3}$ Universidad César Vallejo, PERU
}

Received 19 September 2021 - Accepted 5 December 2021

\begin{abstract}
As part of a recent change, Augmented Reality (AR) has filled engineering classrooms, being employed for various pedagogical purposes around the world. However, little is known about the different features and uses of this technology in Latin America. This Scoping Review asks how are educational AR systems designed, used and evaluated in the region, comparing this to the international literature. To address this question, we charted 36 conference papers and scientific articles, taking care of the quality gaps and methodological diversity within our sample. Our results show that, even though most converge on conventional research, design and pedagogical practices, engineering educators working at institutes are taking the lead of design, pedagogical and research innovation. Furthermore, we show that Latin-American literature distinctively reveals how teachers adapt to the particular contexts of teaching, and the special importance of the usually overlooked conference papers literature.
\end{abstract}

Keywords: augmented reality, engineering education, post-secondary education, Latin America, PRISMA-ScR

\section{INTRODUCTION}

We tend to assume that, for pedagogical purposes, STEM faculties should be the first to adopt current teaching technologies before any other educational institution, as show various summaries of innovations (Hernandez-de-Menendez et al., 2020; Mkrttchian et al., 2019). But specialists seem to overlook the fact that this progressive change depends on a sum of external factors apart from the sole diffusion of innovations, as happens to be the case with $\mathrm{AI}$, e-learning, and data mining (Aljawarneh, 2020; Alyahyan \& Dustegor, 2020; Greenan, 2021; Zawacki-Richter et al., 2019). A case could be made for the inclusion, among these, of the global skill inequalities, which affect higher education in the developing world (Gómez-Tone et al., 2020).

At least since Ivan Sutherland's The Ultimate Display (1965), Augmented Reality (AR), referred as well as "advanced", "improved" and "enriched reality", has been understood as a set of applications that complement or combine real and digital environments, ideally blurring the difference between the two (Billinghurst, 2021; Iatsyshyn et al., 2020). The use of AR in education was first introduced by the aviation industry at the end of the twentieth century, transforming higher education ever since (Akcayır \& Akcayır, 2017; Wang et al., 2018). However, the use of AR in higher education became a protagonist in devoted conferences and publications only during the last five years (Altinpulluk, 2019). As a consequence, numerous recent literature reviews report that the AR literature is filled with evidence-based pedagogical practices and innovative design procedures, benefiting students' motivation and learning (Altinpulluk, 2019; Garzón et al., 2019; Nesenbergs et al., 2021; Sommerauer \& Müller, 2018).

AR seems to be especially useful for STEM education (Ibáñez \& Delgado-Kloos, 2018; Sirakaya \& Sirakaya,

(c) 2022 by the authors; licensee Modestum. This article is an open access article distributed under the terms and conditions of the Creative Commons Attribution License (http://creativecommons.org/licenses/by/4.0/). 


\section{Contribution to the literature}

- We focus on Latin-American publications, quite neglected on earlier reviews.

- Our PRISMA informed Scoping Review notably includes conference papers and scientific articles.

- We build a composite quality index for the quality appraisal of IT case presentations, observational and quasi-experimental studies.

2018, 2020). And since they are usually considered CTML and mobile-learning related technologies, the benefits of $A R^{\prime} s$ environment enhancement for engineering education are well known (Diao \& Shih, 2019; Hernandez-de-Menendez et al., 2020; Singh et al., 2019). But the adoption of AR in STEM teaching still faces some challenges, like the lack of knowledge and skills in teachers, as well as institutional barriers (Barroso Osuna et al., 2019). Additionally, the evidence about the use of $A R$ in the very diverse field of engineering education is still quite unknown in contrast to its applications in other educational disciplines and levels. Considering the existing STEM education disparities in the world (Drew, 2020), we hypothesize that these issues may worsen in Latin-American universities, but there's scant evidence of this.

There are remarkable gaps in the extant literature. Diao and Shih (2019) and Singh et al. (2019) reviewed the research designs, educational outcomes and technological features of AR technologies in journal papers, focusing specifically on Architectural, Civil Engineering and Electronics education. Sirakaya and Sirakaya (2018) performed a similar systematic review including science education and medical training. Other reviews include technologies such as VR (Wang et al., 2020). However, we note a scarcity of variables measured, quality appraisal reports and a general lack of interest in this area of research. Moreover, there is a complete relegation of other literature types as part of these needed technology evaluation synthesis, even though most innovation reports are not published through journal articles. A scoping review under the PRISMA-ScR guidelines seem to be the best choice for an exploratory path.

Hence, this paper addresses the following questions: how are AR systems designed, used and evaluated in engineering education in Latin America, and how does this compares with the rest of the world? To address this question, we present a scoping review of papers and conference articles published by Latin-American authors. To do this, we chart publications from four international databases and perform a threefold quality appraisal according to the different literature types found. We draw inspiration from a wide diversity of contributions: among these, reviews about the use of dynamic and static contents, pedagogical affordances, evaluation types and outcomes of education-oriented AR.

\section{METHOD}

Scoping reviews are comprehensive literature reviews that bring provisional answers to general questions, not requiring the precision of a systematic review (Munn et al., 2018). Previous recent international literature reviews were normally systematic reviews, a few of them being meta-analyses (Garzón et al., 2019) or less systematic methods (Altinpulluk, 2019). This includes a previous scoping review published in this journal (Saltan \& Arslan, 2016), which inspired this work. But, in contrast with the latter, we focus on one particular geographical region and follow the 20 PRISMA-ScR criteria for scoping reviews, proposed originally for literature reviews of medical journals and articles (Tricco et al., 2018).

Scoping reviews under the PRISMA framework proceed by defining research questions, inclusion criteria, search strategies and sources, literature screening, selection, extraction and analysis processes, and result reporting along with discussions (Peters et al., 2020). The protocol for this review was registered in OSF (Bellido García \& Paucar Villacorta, 2021). The complete process is reported in Figure 1. Our research questions were the following:

- What are the main bibliometric patterns of the Latin-American literature reviewed?

- What types of software and hardware systems prefer Latin-American engineering educators employing AR?

- What pedagogical perspectives and practices guide the educational applications of this technology?

- What are the stated advantages and disadvantages of using AR systems in LatinAmerican engineering education?

- What are the research designs in Latin-American tests and evaluations of the said technology?

- Are there significant differences between our results and similar international reviews?

Earlier reviews typically focus on English-written academic papers gathered from sources like SSCI, Scopus, and Google Scholar. We chose to depart from this trend in three ways. First, we selected the databases considering their importance for Latin-American authors: Scielo, the Red Iberoamericana de Información y Conocimiento Científico (REDIB), Web of Science (WOS), and SCOPUS. Secondly, inspired on the recent appraisal 


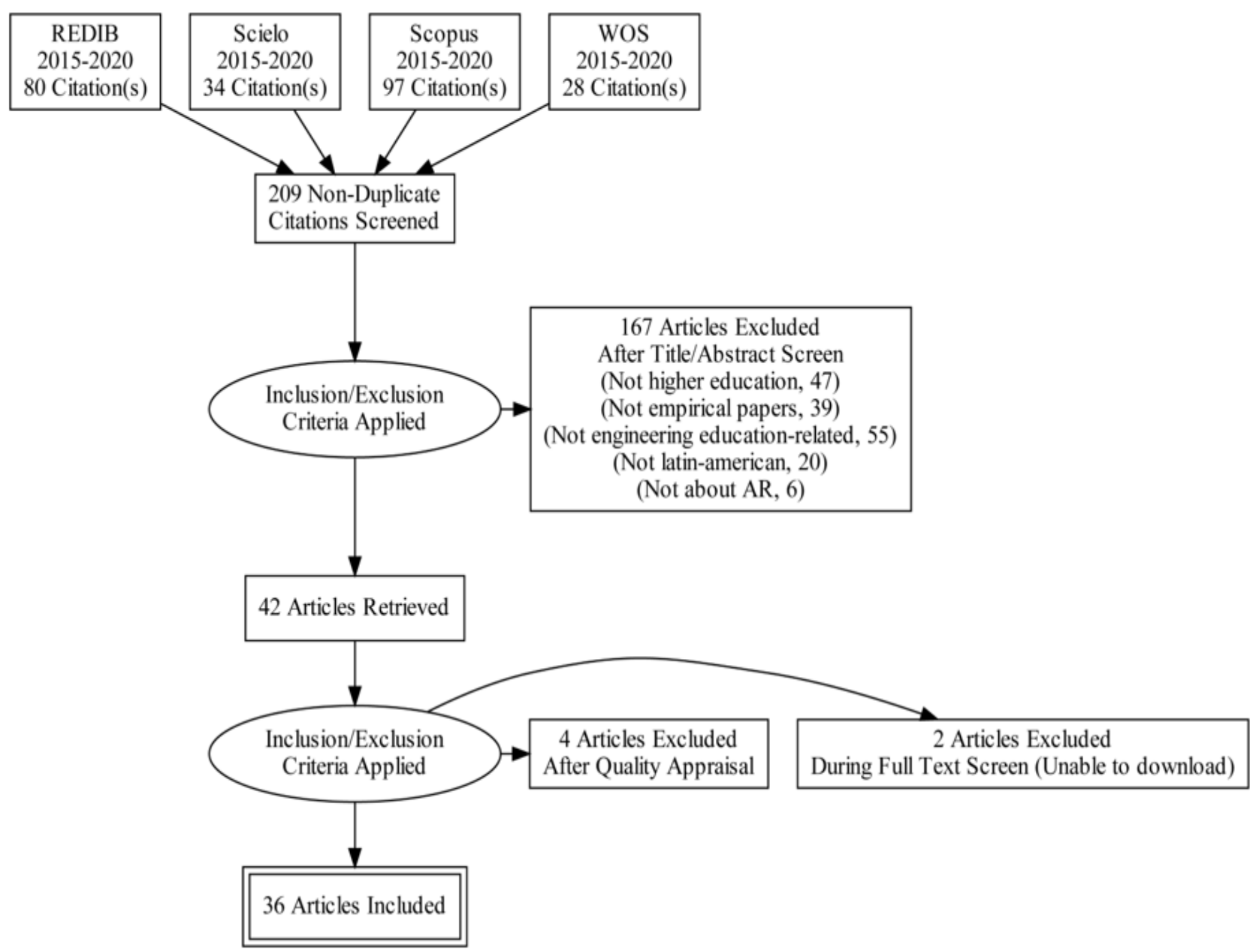

Figure 1. Flow diagram of the scoping review

Table 1. Criteria of inclusion and exclusion recommended by the PRISMA-ScR guidelines

\begin{tabular}{|c|c|c|}
\hline Criteria & Inclusion & Exclusion \\
\hline Context & \multicolumn{2}{|c|}{$\begin{array}{l}\text { Post-secondary educational programs (in general), including work- Papers about the use of AR in non- } \\
\text { based skill training, with a focus on one or many engineering fields educational or work-based skill training } \\
\text { Papers written by authors from the following countries: Mexico, }\end{array}$} \\
\hline Population & $\begin{array}{l}\text { Papers that describe the use of AR by engineering students, } \\
\text { professionals, and teachers in tertiary education institutions } \\
\text { (including technical schools) in Latin America }\end{array}$ & $\begin{array}{l}\text { Papers that solely describe AR applications } \\
\text { by individuals of disciplines other than } \\
\text { engineering specializations, or engineering } \\
\text { related applications in school students, or } \\
\text { students foreign to Latin America }\end{array}$ \\
\hline Concept & $\begin{array}{l}\text { Reports about the use of augmented, mixed, enriched or hybrid } \\
\text { reality that describe the use of AR in tertiary education as a main } \\
\text { goal, accounting for case studies (design) and evaluations }\end{array}$ & $\begin{array}{l}\text { Education technology reports that do not } \\
\text { account for the use of AR at all }\end{array}$ \\
\hline $\begin{array}{l}\text { Types of } \\
\text { evidence } \\
\text { sources }\end{array}$ & $\begin{array}{l}\text { Conference papers and scientific articles that describe empirical } \\
\text { uses of AR in the classroom }\end{array}$ & $\begin{array}{l}\text { Literature reviews, essays, thesis, } \\
\text { dissertations, and book chapters not } \\
\text { interested in the description or evaluation of } \\
\text { an AR application usage by students or } \\
\text { teachers }\end{array}$ \\
\hline Time period & Literature between 2015-2020 & Literature outside this period \\
\hline
\end{tabular}

of grey literature to conduct literature reviews (Adams et al., 2017; Garousi et al., 2019; Hartling et al., 2017), we decided to include conference proceedings and scientific journals in my search. Note that the first were the most numerically dominant in Scopus database searches, despite being usually considered a "weak" form of white formal literature. Finally, my search was intentionally multi-linguistic, spanning to a broad English, Spanish and Portuguese-written literature.

I defined four inclusion and four exclusion criteria, listed in Table 1. The search and duplicate elimination 
Table 2. Search strings used for each database

\begin{tabular}{|c|c|}
\hline Database & Search strings \\
\hline Scopus & 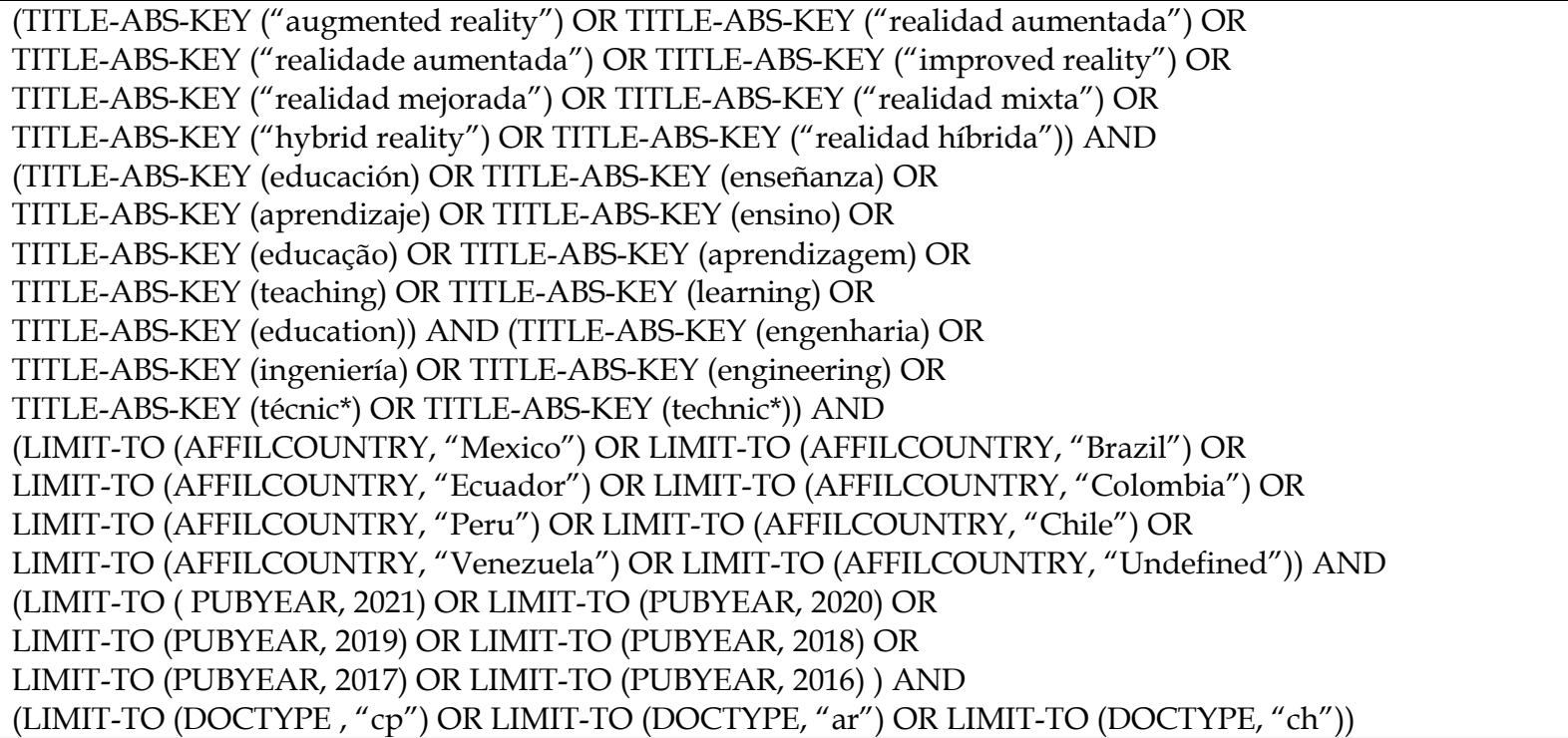 \\
\hline & 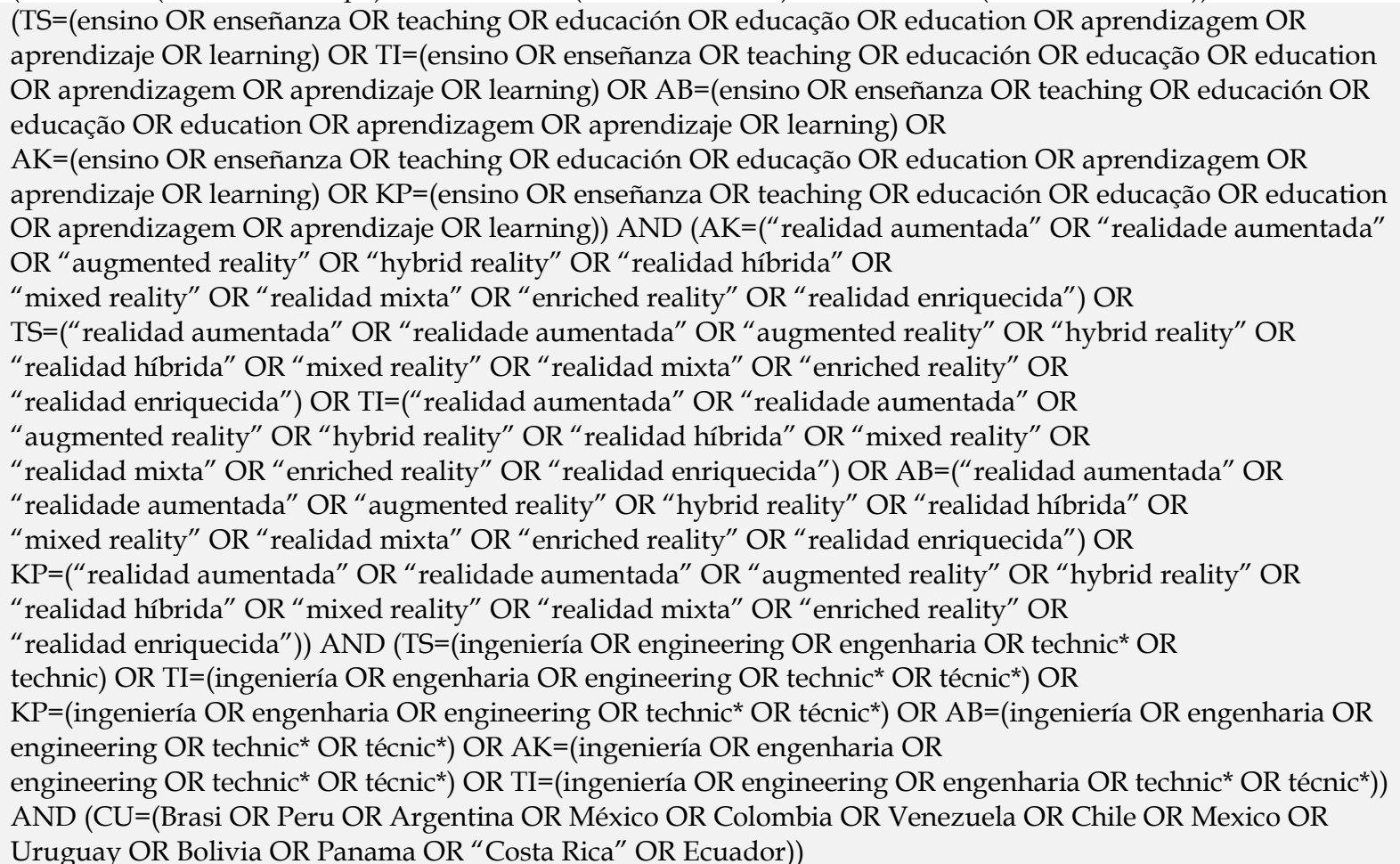 \\
\hline Scielo & $\begin{array}{l}\text { (((ti:(realidad aumentada)) OR (ti:(augmented reality)) OR (ti:(realidade aumentada))) AND } \\
\text { (ingenería) OR (engineering) OR (Engenharia))) }\end{array}$ \\
\hline REDIB & $\begin{array}{l}\text { ("realidad aumentada" OR "augmented reality" OR "realidade aumentada" OR "realidad híbrida" OR "realidad } \\
\text { mixta" OR "mixed reality" OR "hybrid reality") (ingeniería OR engenharia OR engineering) }\end{array}$ \\
\hline
\end{tabular}

process was made during February 2021. The search strings used are shown in Table 2. Scopus and WOS allowed me to be much more specific with my search, and hence produced larger search strings. Abstract screening lasted for one month after the original search of databases, and the quality assessment of the collected evidence lasted for two more months. While this process was made by only one author, eligibility and quality criteria were chosen by debate and consensus after parallel readings of the extant literature.
Judging by the PRISMA-ScR criteria, research quality appraisals are uncommon and considered optional for scoping reviews. However, the authors felt that this review could be affected by the lack of effective peerreview practices in some Latin-American journals. This is supported by the fact that most journals listed in the largest Latin-American publications database, Latindex, don't reach the second quartile of the SCImago Journal Rank. On the other hand, conference papers can easily omit important details due to space limitations, and peer-reviewing before publication isn't usually 
Table 3. Quality appraisal criteria considered by weight and applicability

\begin{tabular}{|c|c|c|c|}
\hline No & Criteria & Weight & Applicable to \\
\hline 1 & $\begin{array}{l}\text { Is there a clear statement (definition) of the aims (goals, purposes, problems, motivations, } \\
\text { objectives, questions) of the research? }\end{array}$ & 1 & All \\
\hline 2 & Is there an adequate description of the context in which the research was carried out? & 1 & All \\
\hline 3 & Does the report answer the research question defined or presents the results in a clear way? & 1 & All \\
\hline 4 & Is the report based on research? & 1 & All \\
\hline 5 & Is the report well-written? & 1 & All \\
\hline 6 & Is there any intention to be a technological innovation? & 1 & All \\
\hline 7 & Is the technological design based on recent innovations? & 1.5 & All \\
\hline 8 & $\begin{array}{l}\text { Does the author succeed in developing a legitimate innovation (e. g., is the software more } \\
\text { useful than already existing software?) }\end{array}$ & 1.5 & All \\
\hline 9 & $\begin{array}{l}\text { Does the author add additional relevant information? (Code, operation steps, common } \\
\text { problems and their resolution) }\end{array}$ & 1.5 & All \\
\hline 10 & Does the report include images representing steps of operation? & 1.5 & All \\
\hline 11 & $\begin{array}{l}\text { Is there an explicit relationship between a pedagogical perspective and the technology } \\
\text { described? }\end{array}$ & 1.5 & All \\
\hline 12 & Are pedagogical concepts informed by recent literature? & 1 & All \\
\hline 13 & Does it add a methodological innovation when evaluating the technology? & 1 & Case+Eval. \\
\hline 14 & Was the simple selection criteria explicitly stated? & 1.5 & Case+Eval. \\
\hline 15 & Does the sample seem representative of the wider population? & 1 & Case+Eval. \\
\hline 16 & Are the sample traits relevant to the report's population? & 1.5 & Case+Eval. \\
\hline 17 & Does it include sample bias/dropout measures? & 1 & Case+Eval. \\
\hline 18 & Does the design answer the research questions? & 1.5 & Case+Eval. \\
\hline 19 & $\begin{array}{l}\text { Is the evaluation design stated with clarity and is there coherence between methodology } \\
\text { and results? }\end{array}$ & 1 & Case+Eval. \\
\hline 20 & Were the measures trustable, validated and equally applied to the whole sample? & 1.5 & Case+Eval. \\
\hline 21 & Is there a good description of the measures used? & 1 & Case+Eval. \\
\hline 22 & Are the results credible (are there other means of verification reported)? & 1.5 & Case+Eval. \\
\hline 23 & Were there only positive results reported? (Check if not) & 1.5 & Case+Eval. \\
\hline 24 & Are the study limitations discussed? & 1.5 & Case+Eval. \\
\hline 25 & Do numerical results answer the study's research question? & 1.5 & Case+Eval. \\
\hline 26 & Is the researcher \& subjects relationship discussed? & 1.5 & Case+Eval. \\
\hline 27 & Was pre/post change measured? & 1 & $\begin{array}{l}\text { Case+Eval.: } \\
\text { Comparative }\end{array}$ \\
\hline 28 & Are individual and group outcomes compared? & 1.5 & $\begin{array}{l}\text { Case+Eval.: } \\
\text { Comparative }\end{array}$ \\
\hline 29 & Are compared groups similar or is there an explicit comparability intention? & 1.5 & $\begin{array}{l}\text { Case+Eval.: } \\
\text { Comparative }\end{array}$ \\
\hline 30 & Are baseline or descriptive data included? & 1 & $\begin{array}{l}\text { Case+Eval.: } \\
\text { Reggression-based }\end{array}$ \\
\hline 31 & Is a control group included? & 1.5 & Cuasiexper. \\
\hline 32 & Is there a discussion about causality? & 1.5 & Cuasiexper. \\
\hline 33 & Is there an explicit mention of randomization? & 1 & Cuasiexper. \\
\hline 34 & Was the randomization method correct? & 1.5 & Cuasiexper. \\
\hline 35 & Was the randomization process blind? & 1.5 & Cuasiexper. \\
\hline 36 & $\begin{array}{l}\text { Was the study double-blinded (is the author intentionally unconscious about which subjects } \\
\text { are assigned to each group)? }\end{array}$ & 1 & Cuasiexper. \\
\hline
\end{tabular}

registered in proceedings. And while the design of new quality criteria could be deemed as a risky task, it is true that various criteria exist (Garousi et al., 2019), and that usually recommended criteria is unsuitable for all types of engineering literature (Kitchenham \& Brereton, 2013).

Hence, we iteratively designed and tested a weighted quality index for each report based on three components: an indicator of the quality of technology design presentations (based on the principles set by Isaksson et al. (2020), Petersen (2020) and Schön et al. (2017)), an indicator of the quality of the empirical testing or evaluation of the technology earlier presented, excluding design-only papers (Liu et al., 2016;
Mårtensson et al., 2019), and an independent indicator of the quality of quasi-experimental designs (drawn mostly from Cochrane criteria). The final criteria list with weights and requirements is shown in Table 3 . The indicators where defined as the division between the sum of weights and the weights of all applicable criteria for the current paper. The second component extended to three additional criteria when the papers where comparative or regression based- designs. All papers below the $40 \%$ threshold in all three indicators at the same time were excluded. The composite index was defined by $\sum \max (i)-\sum(i)$, where $i$ are the existing indicators for the three types of literature, and quartiles 
where calculated as an additional variable for exploratory data analysis.

Following the selection of a final sample of documents $(n=36)$, we automatically extracted bibliographic data using Zotero (database name, author, year, country, publication, item type, accessibility and URL/DOI). We defined thirty-five variables for the chart, divided in four big groups: bibliographic details, research design, AR design features, and pedagogical traits of the AR systems, along with a quality index variable and a final reviewer commentary. Many variables were inspired in earlier reviews; buy the variable "Application type", drawn from Altinpulluk (2019), was simplified to indicate exclusive categories. Only three variables of the second and third group weren't taken from the literature, including the presence of coding tasks, and the origin of 3D models in 3D-based AR applications. Furthermore, we grouped many of these variables, including: journal name, engineering specialization, type of educational institution, type of device, software name, pedagogical perspectives, pros and cons. The full list of variables along with their sources in the literature and examples are shown in Tables 4-9.

Table 4. Data extracted from the literature - Part 1

\begin{tabular}{|c|c|c|c|c|}
\hline Code & $\begin{array}{l}\text { Quality } \\
\text { quartile }\end{array}$ & e & DB & Country \\
\hline [1] & Good & A mobile augmented reality system to support machinery operations in scholar environments & Scopus & Mexico \\
\hline [2] & Weak & $\begin{array}{l}\text { A pilot study on the use of mobile augmented reality for interactive experimentation in quadratic } \\
\text { equations }\end{array}$ & & Mexico \\
\hline [3] & Weak & A relidade aumentadana apresent ação de produtos cartográficos & Scielo & Brazil \\
\hline [4] & Regular & A smartphone-based augmented reality system for university students for learning digital electronics & Scopus & Mexico \\
\hline [5] & Good & Adoção de realidade aumentada no ensino de resistência dos materiais & REDIB & Brazil \\
\hline [6] & Optimal & An education application for teaching robot arm manipulator concepts using augmented reality & Scopus & Mexico \\
\hline [7] & Weak & Aplicación de realidad aumentada para la enseñanza de la robótica & REDIB & Mexico \\
\hline [8] & Weak & olicación móvil conrealidad aumentada para la asignatura de metodología de la investigación & REDIB & Mexico \\
\hline [9] & Good & $\begin{array}{l}\text { Aplicación móvil de realidad aumentada, utilizando la metodología mobile-d, para el entrenamient de } \\
\text { técnicos de mantenimiento de maquinaria pesada en la empresa zamine service peru sac }\end{array}$ & REDIB & Peru \\
\hline [10] & Regular & Arquitectura interactiva como soporte al aprendizaje situ ado en la enseñanza de la ingeniería & WoS & Colombia \\
\hline [11] & Weak & Augmented reality and Matlab® for visuospatial competence development & Scopus & Mexico \\
\hline [12] & Good & $\begin{array}{l}\text { Determining which touch gestures are commonly used when visualizing physics problems in augmented } \\
\text { reality }\end{array}$ & Scopus & Mexico \\
\hline [13] & Good & $\begin{array}{l}\text { Development of an augmented reality environment for the assembly of a precast wood-frame wall using } \\
\text { the BIM model }\end{array}$ & Scielo & Brazil \\
\hline [14] & Weak & $\begin{array}{l}\text { Diseño y desarrollo de un sistema de realidad mixta para la enseñanza-Aprendizaje de la física de } \\
\text { agujeros negros }\end{array}$ & Scopus & Colombia \\
\hline [15] & Weak & $\begin{array}{l}\text { trategia colaborativa en entornos trimensionales como estrategia didáctica de aprendizaje de } \\
\text { tructuras iterativas en programación computacional }\end{array}$ & REDIB & Colombia \\
\hline [16] & Weak & $\begin{array}{l}\text { performance of static and dynamic contents deploye } \\
\text { n }\end{array}$ & Scopus & Colombia \\
\hline [17] & Regular & $\begin{array}{l}\text { Handheld augmented reality system for resistive electric circuits understanding for undergraduate } \\
\text { students }\end{array}$ & Scopus & \\
\hline [18] & Good & 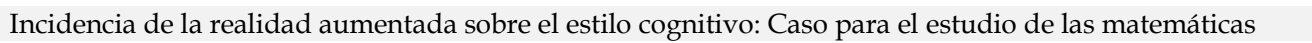 & REDIB & Colombia \\
\hline [19] & Regular & $\begin{array}{l}\text { ternational comparative pilot study of spatial skill development in engineering students through } \\
\text { tonomous augmented reality-based training }\end{array}$ & Scopus & \\
\hline [20] & Optimal & formación de ingenieros en sistemas automotrices mediante la realidad aumentada & REDIB & Mexico \\
\hline [21] & Regular & $\begin{array}{l}\text { MATHPOL: Development of mathematical competencies in engineering students using project-oriented } \\
\text { learning }\end{array}$ & Scopus & Mexico \\
\hline [22] & Regular & $\begin{array}{l}\text { Measurement of emotional variables through a brain-computer interface in the interaction with books } \\
\text { with augmented reality in higher education }\end{array}$ & Scopus & Colombia \\
\hline [23] & ood & {$\left[F_{0}\right.$} & & \\
\hline [24] & Good & & Scopus & \\
\hline [25] & Regular & $\begin{array}{l}\text { Realidad aumentada como herramienta de apoyo al aprendizaje de las funciones } \\
\text { trascendentes }\end{array}$ & WoS & Colombia \\
\hline [26] & Good & 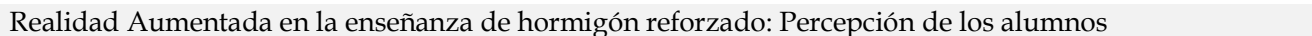 & & \\
\hline [27] & Optimal & $\begin{array}{l}\text { Realidad aumentada: Propuesta metodológica para la didáctica de diseño industrial en el ámbito } \\
\text { universitario }\end{array}$ & REDIB & Chile \\
\hline [28] & Optimal & $\begin{array}{l}\text { Self-learning guide for bioloid humanoid robot assembly with elements of augmented reality to support } \\
\text { experiential learning in sauro research seeding }\end{array}$ & Scopus & Colombia \\
\hline [29] & Optimal & stemas de aprendizaje colaborativo móvil con realidad aumentada & & \\
\hline [30] & Regular & Smart objects for engineering labs: Boosting exploratory learning in higher education & Scopus & Ecuador \\
\hline [31] & Optimal & $\begin{array}{l}\text { Teaching multidisciplinary teams requirements for undergraduate students: An approach to augmented } \\
\text { reality software in design thinking context }\end{array}$ & Scopus & Brazil \\
\hline [32] & Regular & $\begin{array}{l}\text { Um material potencialmente significativo para o ensino da engenharia civil utilizando impressora 3D e } \\
\text { realidade aumentada: Uma experiência com alunos do ensino médio e do ensino su... }\end{array}$ & REDIB & Brazil \\
\hline [33] & Optimal & phenomena & & \\
\hline [34] & & & & \\
\hline [35] & Good & 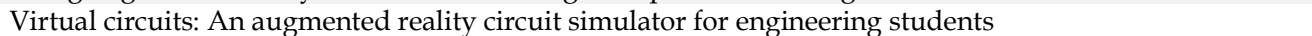 & & Ecuador \\
\hline [36] & Weak & Virtual environment for training oil \& gas industry workers & Scopus & Ecuador \\
\hline
\end{tabular}


Table 5. Data extracted from the literature - Part 2

\author{
Author \\ Monroy Reyes, A., Vergara Villegas, O. O., Miranda \\ Bojórquez, E., Cruz Sánchez, V. G., \& Nandayapa, M. \\ Castillo, R. I. B., Sanchez, V. G. C., \& Villegas, O. O. V. \\ de Oliveira Souza, W., Mira de Espindola, G., Alves \\ Pereira, A. R., \& Marques de Sá, L. A. C. \\ Avilés-Cruz, C., \& Villegas-Cortez, J. \\ Silva, J.; Souza, F. da F. de, Sedraz, L., \& Ramos, J. L. C. \\ Hernández-Ordoñez, M., Nuño-Maganda, M. A., \\ Calles-Arriaga, C. A., Montaño-Rivas, O., \& Bautista \\ Hernández, K. E. \\ Mendoza Pérez, M. A., Cruz Flores, R. G., Villalba \\ Hernández, A. A., Calderón Rodríguez, J. A., \& Patiño, \\ E. A. \\ Soberanes Martín, A., Castillo Mendoza, J. L., \& Peña \\ Martín, A. \\ Gamboa Cruzado, J., Larico Uchamaco, G. R., Soto \\ Soto, L., Chacón Malasquez, N., Tuiro Achulle, J., \& \\ Guzman Chambi, S. C. \\ Gomez, J. E., Hernandez, V., \& Morales, M. \\ Flores-Amado, A., Diliegros-Godines, C. J., Trevino, J. \\ P., Sayeg-Sanchez, G., \& Gonzalez-Hernandez, H. G. \\ del Rio Guerra, M., Martín-Gutiérrez, J., Vargas- \\ Lizárraga, R., \& Garza-Bernal, I. \\ Cuperschmid, A. R. M., Grachet, M. G., \& Fabrício, M. \\ M. \\ Grimaldo, A. C. R., \& Chaparro, E. M. V.
}

Jiménez Toledo, J. A., Collazos Ordoñez, C. A.,

Hurtado Alegría, J. A., \& Pantoja Y, W. L.

Montoya, M. H., Díaz, C. A., \& Moreno, G. A.

Reyes-Aviles, F., \& Aviles-Cruz, C.

Buitrago-Pulido, R. D.

Gómez-Tone, H. C., Martin-Gutierrez, J., Anci, L. V., \&

Luis, C. E. M.

Cortés Caballero, J. M.

Medina Herrera, L. M., Glaros, D., \& Abalo, M. A.

Rojas-Contreras, M., Peña-Cortés, C. A., \& CañasRodríguez, S. M.

De Almeida Carlos, G. A., Ferro, V., Lisboa, R., \& Da Silva, A.

Alvarez-Marin, A., Castillo-Vergara, M., Pizarro-

Guerrero, J., \& Espinoza-Vera, E.

Marquez-Diaz, J. E., \& Morales-Espinosa, L. A.

Nolasco de Almeida Mello, G., \& Cabero Almenara, J.

Laurens Arredondo, L. A.

Lemmel-Vélez, K., \& Valencia-Hernandez, C. A.

Mendoza Morán, V. del R., Rivera Guevara, R., \& Barriga Andrade, J.

Ullón, H., Zambrano, D., \& Domínguez, F.

Almeida, E. M. D., Damasceno, E. F., \& Lrerario, A.

Rodrigues Júnior, A. S., da Costa Gomes, G. J., Cara

Berteges, L. F., de Souza Siqueira Pereira, C., \& de

Alencar Carvalho, C. V.

Morales, A. D., Sanchez, S. A., Pineda, C. M., \&

Romero, H. J.

Ramirez Flores, P. G., Mendoza Medina, J. A., Gonzalez Mendivil, E., \& Villegas Villarreal, A. R.

Lucas, P., Vaca, D., Dominguez, F., \& Ochoa, X.

Garcia, C. A., Naranjo, J. E., Gallardo-Cardenas, F., \& Garcia, M.V.
Year Journal

2016 Computer Applications in Engineering Education

DOI

2015 Mathematical Problems in Engineering

2016 Boletim de Ciências Geodésicas

2019 Computer Applications in Engineering Education

2015 Anais dos Workshops do Congresso Brasileiro de Informática na Educação

2018 Mobile Information Systems

$10.1155 / 2018 / 6047034$

2017 Pistas educativas

10.31876/ie.v1i1.6

2018 Pistas educativas

10.26871/killkana_tecnica. v1i2.78

2017 Ceprosimad

2015 Revista Educacion en Ingenieria

10.26507/rei.v10n20.575

2020 IEEE Global Engineering Education Conference, EDUCON

2018 Lecture Notes in Computer Science

10.1109/EDUCON45650.20 20.9125205

2016 Ambiente Construído

2019 Proceedings of the LACCEI international Multiconference for Engineering, Education and Technology

$10.1155 / 2015 / 946034$ 0.1590/s1982-

21702016000400045

$10.1002 /$ cae. 22102

2015 Revista Investigium Ire: Ciencias Sociales y Humanas 10.15658/CESMAG15.0506 0207

2017 Eurasia Journal of Mathematics, Science and Technology Education

2018 Computer Applications in Engineering Education

2015 Educación y educadores

urasia.2017.0061 $7 \mathrm{a}$

10.1002/cae.21912

10.5294/edu.2015.18.1.2

10.3390/SYM12091401

2016 Edutec. Revista Electrónica de Tecnología Educativa 10.21556/edutec.2016.58.83 8

20202020 5th International Conference on Information Technologies in Engineering Education, Inforino 2020

2020 Journal of Physics: Conference Series

$10.1109 /$ Inforino48376.2020 .9111856

$10.1088 / 1742$

6596/1674/1/012016

2019 Proceedings of the International Conference on Power Electronics and Drive Systems

2017 Formacion Universitaria

10.1109/PEDS44367.2019.8 998825

$10.4067 / \mathrm{S} 0718$

50062017000200005

2019 Revista Educacion en Ingenieria

2020 ALTERIDAD. Revista de Educación

$10.26507 /$ rei.v15n29.1037

$10.17163 /$ alt.v15n1.2020.01

10.18779/ingenio.v1i1.11

$10.1007 / 978-3-030-23528$ $4 \_17$

2019 Communications in Computer and Information Science

2016 Revista Politécnica (Quito)

10.5335/rbca.v12i1.10139

2017 12th Latin American Conference on Learning Objects 10.1109/LACLO.2017.8120 and Technologies, LACLO 2017

915

2019 Proceedings - Frontiers in Education Conference, FIE 10.1109/FIE.2018.8658529

10.34117/bjdv6n3-091

2019 IOP Conference Series: Materials Science and Engineering

$10.1088 / 1757$

$899 \times / 519 / 1 / 012021$

$10.1007 / 978-3-319-73323$ Social-Informatics and Telecommunications Engineering

2018 Proceedings - IEEE 18th International Conference on 10.1109/ICALT.2018.00097 Advanced Learning Technologies, ICALT 2018

2019 Lecture Notes in Computer Science

10.1007/978-3-030-25999032 
Table 6. Data extracted from the literature - Part 3

\begin{tabular}{|c|c|c|c|c|c|c|}
\hline Item Type & Population & Year & Course & Specialization & Educative institution & Research Design \\
\hline Article & $\begin{array}{l}\text { Students \& } \\
\text { teachers }\end{array}$ & & & Mechatronic Engr. & UACJ & Design \& Evaluation \\
\hline Article & $\begin{array}{l}\text { Students \& } \\
\text { teachers }\end{array}$ & 3 & Math & Many & $\begin{array}{l}\text { Universidad Autónoma } \\
\text { Ciudad Juárez }\end{array}$ & Design \& Evaluation \\
\hline Article & Students & 1 & & Cartographic Engr. & $\begin{array}{l}\text { Universidade Federal do } \\
\text { Piauí }\end{array}$ & Design \& Evaluation \\
\hline Article & Students & 1 & & Electronic Engr. & UNAM & Design \& Evaluation \\
\hline Article & Students & & Materials resistance & Engineering & $\begin{array}{l}\text { Universidade Federal do Vale } \\
\text { do São Francisco }\end{array}$ & Evaluation \\
\hline Article & & & & & & Design-only \\
\hline Article & $\begin{array}{l}\text { Students \& } \\
\text { teachers }\end{array}$ & 5 & Advanced Robotics & Computation Engr. & UAEM Valle del Chalco & Design-only \\
\hline Article & $\begin{array}{l}\text { Students \& } \\
\text { teachers }\end{array}$ & & Research Methodology & Computation Engr. & UAEMEX & Design \& Evaluation \\
\hline Article & Employees & & $\begin{array}{l}\text { Unnamed Training } \\
\text { Workshop }\end{array}$ & & Zamine Service Perú SAC & Design \& Evaluation \\
\hline Article & Students & 1 & $\begin{array}{l}\text { Introduction to } \\
\text { Engineering }\end{array}$ & Many & Universidad de Córdoba & Design \& Evaluation \\
\hline Conference Paper & Students & 2 & Math III & Engineering (many) & Tecnológico de Monterrey & Design \& Evaluation \\
\hline Conference Paper & Students & 1 & Physics & Mechanical Engr. & Universidad de Monterrey & Design \& Evaluation \\
\hline Article & Students & & & Civil Engr. & Universidade de São Paulo & Design \& Evaluation \\
\hline Conference Paper & Students & many & $\begin{array}{l}\text { Black holes and time } \\
\text { machines (short course) }\end{array}$ & & $\begin{array}{l}\text { Universidad Nacional de } \\
\text { Colombia }\end{array}$ & Design-only \\
\hline Article & Students & 1 & Many (coding) & System Engr. & CESMAG/UAN & Design \& Evaluation \\
\hline Article & Students & 1 & Electronics basics & Electronic Engr. & $\begin{array}{l}\text { Institución Universitaria } \\
\text { Salazar y Herrera }\end{array}$ & Design \& Evaluation \\
\hline Article & Students & 1 & Electronics basics & Electronic Engr. & $\begin{array}{l}\text { Universidad Aútonoma } \\
\text { Metropolitana }\end{array}$ & Design \& Evaluation \\
\hline Article & Students & 2 & Vectorial calculus & Industrial Engr. & $\begin{array}{l}\text { Escuela Colombiana de } \\
\text { Carreras Industriales }\end{array}$ & \\
\hline Article & Students & 1 & Graphic engineering & Many & UCSP(Perú), ULL(España) & Evaluation-only \\
\hline Article & & & & Automobile Engr. & Instituto Politécnico Nacional & Design-only \\
\hline Conference Paper & $\begin{array}{l}\text { Students \& } \\
\text { teachers }\end{array}$ & & Calculus & Many & Tecnológico de Monterrey & Design \& Evaluation \\
\hline Conference Paper & Students & & $\begin{array}{l}\text { Algorithms and } \\
\text { structures }\end{array}$ & System Engr. & Universidad de Pamplona & Design \& Evaluation \\
\hline Conference Paper & Students & & Power electronics & Electronic Engr. & instituto Federal de Alagoas & Design-only \\
\hline Article & Students & & Fluid Mechanics & Civil Industrial Engr. & Universidad de La Serena & Design \& Evaluation \\
\hline Article & Students & & Calculus I & System Engr. & $\begin{array}{l}\text { Universidad de } \\
\text { Cundinamarca }\end{array}$ & Design \& Evaluation \\
\hline Article & Students & 4 & Reinforced Concrete & Civil Engr. & $\begin{array}{l}\text { Pontificia Universidad } \\
\text { Católica de Minas Gerais }\end{array}$ & Design \& Evaluation \\
\hline Article & Students & & Many (Structural Design) & Civil Industrial Engr. & $\begin{array}{l}\text { Universidad Católica de } \\
\text { Maule }\end{array}$ & Design-only \\
\hline Conference Paper & Students & & & Mechatronic Engr. & $\begin{array}{l}\text { Institución Universitaria } \\
\text { Pascual Bravo }\end{array}$ & Design-only \\
\hline Article & Students & & & System Engr. & Universidad de Guayaquil & \\
\hline Conference Paper & Students & 1 & Physics I & Many & $\begin{array}{l}\text { Escuela Superior Politecnica } \\
\text { del Litoral }\end{array}$ & Design \& Evaluation \\
\hline Conference Paper & Students & & Systems analysis & Software Engr. & $\begin{array}{l}\text { Universidad Tecnológica } \\
\text { Federal de Paraná }\end{array}$ & Design \& Evaluation \\
\hline Article & Students & 1 & Many (Structural Design) & Engr. & Universidade de Vassouras & Design \& Evaluation \\
\hline Conference Paper & & & Mechanical physics & Many & Corposucre & Design \& Evaluation \\
\hline Conference Paper & Students & & Reading (short course) & Many & Tecnológico de Monterrey & Design-only \\
\hline Conference Paper & Students & 3 & Electrical Circuit Analysis & Electric Engr. & $\begin{array}{l}\text { Escuela Superior Politecnica } \\
\text { del Litoral }\end{array}$ & Design \& Evaluation \\
\hline Conference Paper & Employees & & $\begin{array}{l}\text { HART object Training } \\
\text { Workshop }\end{array}$ & & $\begin{array}{l}\text { Empresa de Petróleo y Gas } \\
\text { (no dice) }\end{array}$ & Design-only \\
\hline
\end{tabular}


Table 7. Data extracted from the literature - Part 4

\begin{tabular}{|c|c|c|c|c|c|c|c|c|}
\hline $\begin{array}{l}\text { Evaluation } \\
\text { design }\end{array}$ & $\begin{array}{l}\text { Data Collection } \\
\text { technique }\end{array}$ & Outcome variable & $\begin{array}{l}\text { Sample } \\
\text { Size }\end{array}$ & Coding & $\begin{array}{l}\text { Input (Sirakaya } \\
\text { \& Sirakaya) }\end{array}$ & $\begin{array}{l}\text { Software } \\
\text { name }\end{array}$ & Device & App type \\
\hline $\begin{array}{l}\text { Observational } \\
\text { Pre/Pos }\end{array}$ & Survey & Satisfaction \& Performance & 16 & Yes & Labels & Vuforia & VR & Video-based \\
\hline Case study & Questionnaire & $\begin{array}{l}\text { Academic Achievement, } \\
\text { Satisfaction \& Performance }\end{array}$ & 59 & Yes & Labels & Vuforia & $\begin{array}{l}\text { Smartphone \& } \\
\text { Tablets }\end{array}$ & Simulation-based \\
\hline $\begin{array}{l}\text { Observational } \\
\text { Pre/Pos }\end{array}$ & Questionnaire & Satisfaction & 32 & No & Layers & Aumentary & PC & 3D-image based \\
\hline $\begin{array}{l}\text { Observational } \\
\text { Pre/Pos }\end{array}$ & Survey & Satisfaction & 80 & Yes & $\begin{array}{l}\text { Object } \\
\text { recognition }\end{array}$ & Own & Smartphone & Text-Based \\
\hline $\begin{array}{l}\text { Observational } \\
\text { Pre/Pos }\end{array}$ & Questionnaire & Satisfaction & 50 & No & Labels & Aurasma & Tablet & Text-Based \\
\hline Case study & & & & Yes & Labels & ARToolkit & Smartphone & Simulation-based \\
\hline $\begin{array}{l}\text { Observational } \\
\text { Pre/Pos }\end{array}$ & Interviews & Academic achievement & 52 & No & Labels & $\begin{array}{l}\text { Aumentaty } \\
\text { Author }\end{array}$ & $\mathrm{PC}$ & Video-based \\
\hline $\begin{array}{l}\text { Observational } \\
\text { Pre/Pos }\end{array}$ & Survey & $\begin{array}{l}\text { Academic achievement \& } \\
\text { Performance }\end{array}$ & 51 & Yes & Labels & Unity & Smartphone & Text-Based \\
\hline $\begin{array}{l}\text { Quasi- } \\
\text { experiment }\end{array}$ & Observation & KPIs & 30 & Yes & Labels & Vuforia & Smartphone & Text-Based \\
\hline $\begin{array}{l}\text { Observational } \\
\text { Pre/Pos }\end{array}$ & Test & Academic achievement & 40 & No & Labels & Flartoolkit & $\begin{array}{l}\text { Smartphone \& } \\
\text { Tablets }\end{array}$ & 3D-image based \\
\hline $\begin{array}{l}\text { Quasi- } \\
\text { experiment }\end{array}$ & Test & Academic achievement & 56 & Yes & Labels & Own & Smartphone & 3D-image based \\
\hline $\begin{array}{l}\text { Observational } \\
\text { Pre/Pos }\end{array}$ & Interviews & Satisfaction & 26 & Yes & Labels & Vuforia & Smartphone & 3D-image based \\
\hline $\begin{array}{l}\text { Observational } \\
\text { Pre/Pos }\end{array}$ & Survey \& Test & Satisfaction & 28 & No & $\begin{array}{l}\text { Other (detector } \\
\text { test) }\end{array}$ & Metaio & $\begin{array}{l}\text { Smartphone \& } \\
\text { VR }\end{array}$ & Simulation-based \\
\hline Case study & & & & Yes & Labels & Vuforia & Smartphone & Video-based \\
\hline $\begin{array}{l}\text { Quasi- } \\
\text { experiment }\end{array}$ & Test & Academic achievement & 91 & Yes & Layers & & Smartphone & Simulation-based \\
\hline $\begin{array}{l}\text { Observational } \\
\text { Pre/Pos }\end{array}$ & Survey \& Test & $\begin{array}{l}\text { Academic achievement \& } \\
\text { Satisfaction }\end{array}$ & 16 & Yes & Labels & Vuforia & Smartphone & 3D-image based \\
\hline Case study & $\begin{array}{l}\text { Observation \& } \\
\text { Survey }\end{array}$ & Satisfaction \& Performance & 30 & Yes & $\begin{array}{l}\text { Object } \\
\text { recognition }\end{array}$ & Own & Smartphone & Text-Based \\
\hline $\begin{array}{l}\text { Quasi- } \\
\text { experiment }\end{array}$ & Test & Academic achievement & 83 & Yes & & Arvirtual & Smartphone & 3D-image based \\
\hline $\begin{array}{l}\text { Quasi- } \\
\text { experiment }\end{array}$ & Survey & Habilidad espacial & 312 & Yes & Labels & Own & $\begin{array}{l}\text { PC \& } \\
\text { Smartphone }\end{array}$ & 3D-image based \\
\hline Case study & & & & Yes & Labels & Vuforia & Smartphone & 3D-image based \\
\hline $\begin{array}{l}\text { Observational } \\
\text { Pre/Pos }\end{array}$ & Survey & $\begin{array}{l}\text { Academic achievement \& } \\
\text { Performance }\end{array}$ & 239 & Yes & Layers & Own & $\begin{array}{l}\text { Tablet \& } \\
\text { Smartphone }\end{array}$ & Object Modelling \\
\hline $\begin{array}{l}\text { Observational } \\
\text { comparative }\end{array}$ & Emotiv Insight & Emotions & 5 & No & Labels & & Smartphone & \\
\hline Case study & & & & Yes & Labels & Vuforia & Smartphone & Simulation-based \\
\hline $\begin{array}{l}\text { Observational } \\
\text { Pre/Pos }\end{array}$ & Survey & Satisfaction & 61 & No & Labels & $\begin{array}{l}\text { Vuforia/Uni } \\
\text { ty }\end{array}$ & $\begin{array}{l}\text { Smartphone \& } \\
\text { Tablet }\end{array}$ & 3D-image based \\
\hline Case study & Survey & Satisfaction & 60 & No & Labels & Vuforia & $\begin{array}{l}\text { Smartphone \& } \\
\text { Tablets }\end{array}$ & Text-Based \\
\hline $\begin{array}{l}\text { Observational } \\
\text { Pre/Pos }\end{array}$ & Survey & Satisfaction & 18 & No & Labels & Sketchfab & Smartphone & 3D-image based \\
\hline $\begin{array}{l}\text { Observational } \\
\text { Pre/Pos }\end{array}$ & Test & & & Yes & Labels & Aumentary & $\begin{array}{l}\text { PC \& } \\
\text { Smartphone }\end{array}$ & 3D-image based \\
\hline Case study & & & & & Labels & Build AR Pro & PC & 3D-image based \\
\hline Case study & & & & Yes & Labels & AndroidIM & Smartphone & Location-based \\
\hline $\begin{array}{l}\text { Quasi- } \\
\text { experiment }\end{array}$ & $\begin{array}{l}\text { Questionnaire } \\
\text { \& test }\end{array}$ & $\begin{array}{l}\text { Academic achievement \& } \\
\text { Satisfaction }\end{array}$ & 40 & Yes & Labels & Vuforia & Smartphone & Text-Based \\
\hline $\begin{array}{l}\text { Quasi- } \\
\text { experiment }\end{array}$ & $\begin{array}{l}\text { Questionnaire } \\
\text { \& test }\end{array}$ & Satisfaction & 30 & & & & & \\
\hline $\begin{array}{l}\text { Observational } \\
\text { Pre/Pos }\end{array}$ & Survey & $\begin{array}{l}\text { Academic achievement \& } \\
\text { Performance }\end{array}$ & 30 & No & Labels & Augment & Smartphone & Object Modelling \\
\hline Case study & & Performance & & Yes & Labels & Vuforia & Smartphone & Simulation-based \\
\hline Case study & & & & No & $\begin{array}{l}\text { Other (detector } \\
\text { test) }\end{array}$ & Vuforia & Smartphone & Game-based \\
\hline $\begin{array}{l}\text { Observational } \\
\text { Pre/Pos }\end{array}$ & Questionnaire & Satisfaction & 100 & Yes & Labels & Vuforia & Smartphone & 3D-image based \\
\hline Case study & & & & Yes & $\begin{array}{l}\text { Other (detector } \\
\text { test) }\end{array}$ & Unity & $\begin{array}{l}\text { Smartphone \& } \\
\text { VR }\end{array}$ & Simulation-based \\
\hline
\end{tabular}


Table 8. Data extracted from the literature - Part 5

\begin{tabular}{|c|c|c|c|c|c|c|c|}
\hline $\begin{array}{l}\text { Static / } \\
\text { Dynamic } \\
\text { (Montoya } \\
\text { et al) }\end{array}$ & $\begin{array}{l}\text { Materials } \\
\text { (Chubukova \& } \\
\text { Ponomarenko) }\end{array}$ & $\begin{array}{l}\text { Pedagogical } \\
\text { Perspective (Wu et al.) }\end{array}$ & $\begin{array}{l}\text { Focus } \\
\text { (Wu et } \\
\text { al.) }\end{array}$ & $\begin{array}{l}\text { Evaluation Strategy } \\
\text { (Diaio) }\end{array}$ & 3D-Object Type & Usage (Diaio) & $\begin{array}{l}\text { Interaction } \\
\text { (Belén et al.) }\end{array}$ \\
\hline Dynamic & Skill training & CTML & Tasks & Problem Resolution & $\begin{array}{l}\text { Forms created by } \\
\text { teacher }\end{array}$ & General (graphics, text) & Perception \\
\hline Dynamic & Modelling & Situated Learning & Tasks & Problem Resolution & & General (graphics, text) & Manipulation \\
\hline Static & Modelling & Experiential Learning & Tasks & Problem Resolution & $\begin{array}{l}\text { Forms created by } \\
\text { teacher }\end{array}$ & General (graphics, text) & Perception \\
\hline Static & Modelling & Mobile learning & Tasks & Problem Resolution & & Espec. Aplic. (design, etc.) & Perception \\
\hline Static & Skill training & Mobile learning & Tasks & Problem Resolution & & General (graphics, text) & Perception \\
\hline Dynamic & Modelling & Experiential Learning & Tasks & Problem Resolution & & Espec. Aplic. (design, etc.) & Manipulation \\
\hline Dynamic & Skill training & CTML & Tasks & Problem Resolution & & General (graphics, text) & Perception \\
\hline Static & Skill training & CTML & Tasks & | Problem Resolution & & General (graphics, text) & Manipulation \\
\hline Static & Skill training & Mobile learning & Tasks & Problem Resolution & & General (graphics, text) & Perception \\
\hline Static & Skill training & Situated Learning & Tasks & Problem Resolution & $\begin{array}{l}\text { Forms created by } \\
\text { teacher }\end{array}$ & General (graphics, text) & Manipulation \\
\hline Static & Skill training & CTML & Tasks & Problem Resolution & & General (graphics, text) & Perception \\
\hline Static & Skill training & Experiential Learning & Tasks & Problem Resolution & $\begin{array}{l}\text { Forms created by } \\
\text { teacher }\end{array}$ & General (graphics, text) & Manipulation \\
\hline Dynamic & Modelling & Experiential Learning & Locations & S Personal Project & $\begin{array}{l}\text { Forms created by } \\
\text { teacher }\end{array}$ & Espec. Aplic. (design, etc.) & Manipulation \\
\hline Dynamic & Modelling & Mobile learning & Tasks & Problem Resolution & $\begin{array}{l}\text { Forms created by } \\
\text { teacher }\end{array}$ & General (graphics, text) & Perception \\
\hline Static & Game alike & Mobile learning & Roles & Peer-work & $\begin{array}{l}\text { Forms created in } \\
\text { class }\end{array}$ & Espec. Aplic. (design, etc.) & Perception \\
\hline Dynamic & Modelling & CTML & Tasks & Problem Resolution & $\begin{array}{l}\text { Forms created by } \\
\text { teacher }\end{array}$ & General (graphics, text) & Annotation \\
\hline Dynamic & Modelling & CTML & Tasks & Problem Resolution & & Espec. Aplic. (design, etc.) & Annotation \\
\hline Dynamic & Modelling & CTML & Tasks & Problem Resolution & & General (graphics, text) & Perception \\
\hline Static & $\begin{array}{l}\text { Textbook / } \\
\text { manual }\end{array}$ & CTML & Tasks & Predefined forms & $\begin{array}{l}\text { General } \\
\text { (graphics, text) }\end{array}$ & Manipulation & Acquisition \\
\hline Dynamic & $\begin{array}{l}\text { Textbook / } \\
\text { manual }\end{array}$ & CTML & Roles & $\begin{array}{l}\text { Forms created in } \\
\text { class }\end{array}$ & $\begin{array}{l}\text { General } \\
\text { (graphics, text) }\end{array}$ & Perception & Acquisition \\
\hline Static & $\begin{array}{l}\text { Object } \\
\text { Modelling }\end{array}$ & Collaborative learning & Tasks & Proyecto grupal & $\begin{array}{l}\text { Forms created in } \\
\text { class }\end{array}$ & General (graphics, text) & Manipulation \\
\hline Static & $\begin{array}{l}\text { Textbook / } \\
\text { manual }\end{array}$ & CTML & Tasks & Predefined forms & $\begin{array}{l}\text { General } \\
\text { (graphics, text) }\end{array}$ & Perception & Acquisition \\
\hline Dynamic & Modelling & CTML & Tasks & Problem Resolution & & Espec. Aplic. (design, etc.) & Perception \\
\hline Static & Skill training & Mobile learning & Tasks & Problem Resolution & $\begin{array}{l}\text { Forms created by } \\
\text { teacher }\end{array}$ & General (graphics, text) & Perception \\
\hline Static & Modelling & Mobile learning & Tasks & Problem Resolution & & Espec. Aplic. (design, etc.) & Manipulation \\
\hline Static & Skill training & CTML & Tasks & Problem Resolution & Predefined forms & General (graphics, text) & Perception \\
\hline Static & Skill training & CTML & Tasks & Personal Project & $\begin{array}{l}\text { Forms created in } \\
\text { class }\end{array}$ & General (graphics, text) & Manipulation \\
\hline Static & $\begin{array}{l}\text { Textbook / } \\
\text { manual }\end{array}$ & Situated Learning & Roles & Peer-work & $\begin{array}{l}\text { Forms created by } \\
\text { teacher }\end{array}$ & Espec. Aplic. (design, etc.) & Manipulation \\
\hline Dynamic & Game alike & Situated Learning & Locations & $\begin{array}{l}\text { SGroup-work } \\
\text { sincrónico }\end{array}$ & & General (graphics, text) & Annotation \\
\hline \multirow[t]{2}{*}{ Dynamic } & Modelling & Experiential Learning & Tasks & Peer-work & & General (graphics, text) & Perception \\
\hline & & & & & $\begin{array}{l}\text { Forms created by } \\
\text { teacher }\end{array}$ & Espec. Aplic. (design, etc.) & Annotation \\
\hline Dynamic & $\begin{array}{l}\text { Object } \\
\text { Modelling }\end{array}$ & Experiential Learning & Tasks & Personal Project & $\begin{array}{l}\text { Forms created in } \\
\text { class }\end{array}$ & Espec. Aplic. (design, etc.) & Manipulation \\
\hline Dynamic & Modelling & Mobile learning & Tasks & Problem Resolution & $\begin{array}{l}\text { Forms created by } \\
\text { teacher }\end{array}$ & General (graphics, text) & Annotation \\
\hline Static & Game alike & Situated Learning & Locations & $\begin{array}{l}\text { Group-work } \\
\text { sincrónico }\end{array}$ & $\begin{array}{l}\text { Forms created by } \\
\text { teacher }\end{array}$ & Espec. Aplic. (design, etc.) & Annotation \\
\hline Static & Modelling & Experiential Learning & Tasks & Peer-work & & General (graphics, text) & Manipulation \\
\hline Static & Skill training & Mobile learning & Locations & Problem Resolution & & General (graphics, text) & Manipulation \\
\hline
\end{tabular}


Table 9. Data extracted from the literature - Part 6

\begin{tabular}{|c|c|c|c|}
\hline $\begin{array}{l}\text { Affordances } \\
\text { (Saltan/Arslan) }\end{array}$ & Pros & Cons & Comment \\
\hline Acquisition & Robust, important and looks good & $\begin{array}{l}\text { There are problems with VR view monoscopic (vs. } \\
\text { Stereoscopic); inability to use by more than one } \\
\text { person }\end{array}$ & \\
\hline $\begin{array}{l}\text { Concept } \\
\text { development }\end{array}$ & It is intuitive and motivating for students & $\begin{array}{l}\text { Endogenous design problems are major, "difficult to } \\
\text { handle" }\end{array}$ & $\begin{array}{l}\text { Nice presentation of } \\
\text { results }\end{array}$ \\
\hline
\end{tabular}

development cartography are many and still neglected

Concept Useful and motivating, innovative use

development

Concept

AR fundamental to learning

Many students said that RA is not

Reinforcement

Acquisition

Acquisition

Improves attention

Acquisition

Knowledge and motivation

pedagogical and technological aspects

Concept

development

Increases understanding, time is reduced, KPIs

Acquisition

are met of thecompany

superior performance

It is difficult to prepare all materials

poor evaluation

Design AR?

Pedagogical?

Concept

Reinforcement

Concept

It's better than a software images

।

Tasks have to do

with cars

Students like the application

small sample, not all hand gestures were scheduled

development

Acquisition

Much better than using paper and PC

The image is moved as it is too much updated;

sometimes it does not correspond to the actual image

$\begin{array}{ll}\begin{array}{l}\text { Acquisition } \\ \begin{array}{l}\text { Concept } \\ \text { development }\end{array}\end{array} & \text { contents } \\ \begin{array}{l}\text { Concept } \\ \text { development }\end{array} & \text { The parendizaje is facilitated by dynamic content }\end{array}$

Very good

development

\section{Concept}

development

Low recognition efficiency different shades of

Acquisition

light

AR can be adapted to the needs of learning

styles, and improvement in all notes

Acquisition

It helps a lot and have acceptance

Neceista complemented by other measuring instruments

It is particularly positive for the dependents of the Very good

field and those that are planned before working

Does not eliminate local differences in skills

regarding educational systems

Are questions about the static and dynamic content

Acquisition

Acquisition

Acquisition

Useful in forming

Develop a book in another publication Very good

AR increases integration with reality, relaxation and interest of students

Acquisition

AR serves to remind students of concepts, but

used in conjunction with other modules and tools

Concept The image helps the understanding of fluid

Reinforcement mechanics through better visualization

Concept Students feel great satisfaction with AR

development

Concept

development

Concept

Reinforcement

Acquisition

Very popular among students

Helps motivation, there is free software

Learning is easier for students and increases interest

Concept

Reinforcement

Acquisition

Concept

development

Concept

development

Acquisition

Concept

development

Concept

development

Acquisition

Collaboration between students meet objectives achieved in a different way

great satisfaction

great satisfaction

There is a percentage of them do not feel that RA

helped at all

The phone takes to process many items to lavez;

stable internet connection required

RA is not compatible with old Smartphones, free

software always requires internet

Good summary of

literature

It has literature

review

The difference in ratings is not statistically significant

They could not identify either the data requirements Very bad

Useful in forming

poor evaluation

Accessible for students to come in mid-range

phones

It is hilarious

Ease, educational value and accessibility to

complex concepts

Specially appreciated by young workers and trainees

Speed display GUI

hunting program

books

Older workers do not feel big difference. 


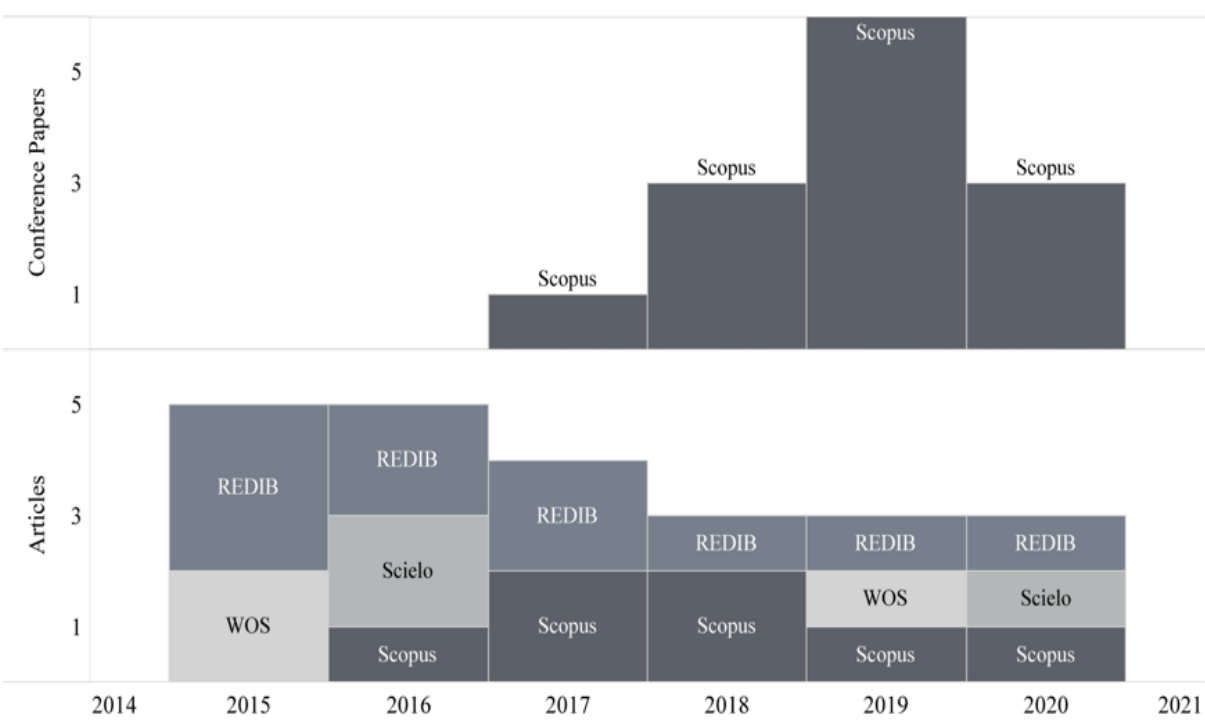

Figure 2. Number of documents by type and data-source per year

The results described below where obtained after exploratory data analysis and visualization during the last month of this research. We decided to add to this analysis the quality variable to minimize our bias against the supposed bad quality of Latin-American research, as stated in PRISMA guidelines. We carefully chose the most telling results, given the space limitations; however, we specifically compare our results with those in other reviews on the subject. We later summarize and interpret these findings within the bigger framework of education technology in the discussion.

\section{RESULTS}

\section{Bibliometric Patterns}

It is usually thought that Brazil is the Latin-American country with the largest scientific productivity in the region, given the prominence of Brazilian authors and journals in Scopus (UNESCO, 2021). However, the largest part of the documents reviewed were written by Mexican ( $\mathrm{n}=12,33 \%)$, Colombian $(\mathrm{n}=9,25 \%)$ and Brazilian ( $\mathrm{n}=7,19 \%)$ authors. Ecuadorian, Chilean and Peruvian authors only authored 8 of the 36 reports (22\%). Furthermore, we noticed that Brazil was the country with the least percentage of documents in Scopus, while the opposite happened with Mexico. This outstanding fact was also found in international reviews that give importance to less science-productive countries than the US or the UK in the pedagogical AR usage-related literature, like Taiwan and Spain (Altinpulluk, 2019; Diao \& Shih, 2019).

The retrieved documents were usually published each in a different journal or conference proceedings book. Computer Applications in Engineering Education, Lecture Notes in Computer Science, Pistas educativas and Revista Educación en Ingeniería were the only publications with at least two documents from the sample. In contrast, previous reviews found that most of the related literature in the world was published in Computers $\mathcal{E}$ Education, The Journal of Science Education and Technology, EURASIA Journal of Mathematics Science and Technology Education, Education Technology and Society and Computers in Human Behavior, among others (Bacca et al., 2014; Iatsyshyn et al., 2020). In contrast, $50 \%$ of our sample was found in journals or proceedings primarily published in Spanish or Portuguese. Among the rest, just one paper was published in the third of the before listed journals.

Our sample seems to have been progressively accumulating in the span between 2015-2020, following the international trend (Altinpulluk, 2019; Diao \& Shih, 2019; Ibáñez \& Delgado-Kloos, 2018). However, we notice a delay in the productivity peak: Even though Altinpulluk (2019) shows an increasing rate of production during 2013-2016 and Diao and Shih (2019) between 2017-2018, we only found a notorious increase in the number of Latin-American documents between 2018-2019. Interestingly, this was driven by a numerical increase of documents from subscription-based journals indexed in Scopus, whereas open-access documents stagnated within the five-year period (except for the REDIB documents, that are decreasing in number versus new Scopus open-access documents). This pattern seems important, given that the extant literature usually rely on WOS or Scopus only. Figure 2 shows the number of documents by type and data-source per year.

Nonetheless, we believe that this change was rather related with an increase in the number of Scopusindexed international conference papers. In fact, the number of papers published in peer-reviewed journals stagnated since 2018 at a rate of three papers per year. It is usually thought that the first are texts of lesser quality than the latter. Overall, we found six documents located in the first ("optimal") quartile of our quality index, and remaining quartiles contained ten documents each. The 

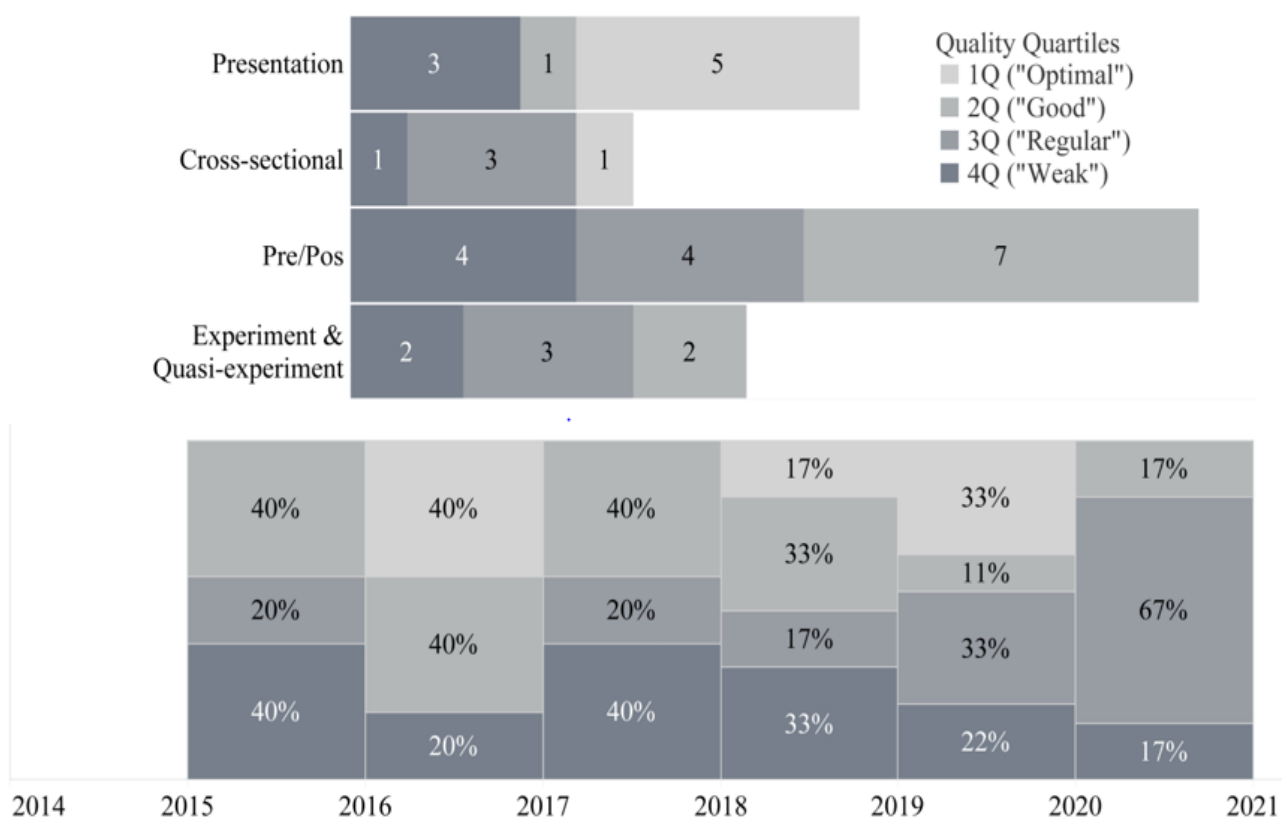

Figure 3. Number (above) and percentage (below) of research quality quartiles by research design and time

number of documents in the third quartile seems to be increasing with time, and in parallel, the fourth and first quartiles shrink. However, we report no relevant differences between the quality of documents screened from different databases or publication types. This finding contrasts with the current selection practices in other reviews, which seem to be guided by an exclusion bias.

\section{Research Designs}

Though some of the works reviewed are simple fulltext descriptions of the design process of technologies or classroom activities $(n=9,25 \%)$, most include some form of empirical evaluation or testing, either by observational $(n=5,14 \%)$, pre/post $(n=15,42 \%)$ or quasiexperimental $(n=7,19 \%)$ designs. Two documents ([5], [19]) are only evaluations. Questionnaires $(n=13,36 \%)$, academic grades $(n=4,11 \%)$ and a mix of both $(n=4,11 \%)$ compose the largest part of data collection techniques used, although some documents also mention qualitative techniques as interviews and observational forms $(n=4,11 \%)$, two mention object recognition data ([4], [17]), one mentions Emotiv Insight cognitive sensory data ([22]) and another one system development outputs ([33]). In contrast, Diao and Shih (2019) find predominantly experimental designs in their engineering-themed review; the further importance of mixed methods and questionnaires for data collection was revealed by the wider reviews of Bacca et al. (2014) and Altinpulluk (2019). Figure 3 depicts the number and percentage of research quality quartiles by research design and time.

The authors in our review mainly engaged with engineering Students $(n=26,72 \%)$, a mix of Students and Teachers $(n=5,14 \%)$ and Employees $(n=2,6 \%)$. The subjects of these studies where systems $(n=7)$, civil $(n=4)$, mechatronics $(n=2)$, and cartographic $(n=2)$ engineering university students, as well as electric $(n=1)$ and industrial $(n=1)$ engineering institute students, and mechanical $(n=3)$, electronic $(n=4)$, and mixed $(n=8)$ engineering specializations students from various institutions. Confirming a wider pattern in the secondary literature, sample sizes in evaluations ranged from 5 [22] to 312 ([19]) subjects, but $55 \%$ of the evaluations fell between 30-60 subjects (e. g., Bacca et al. (2014) found most of the samples in their population to be between 30-200 subjects, while Sirakaya and Sirakaya (2018) placed the sample mean between 31-100 subjects). Besides this, we registered the educational year corresponding to subjects or programs as described in the literature, when possible $(n=16)$.

As shown in Figure 4, most authors worked with first-year students, but older students were also part of bigger sample sizes. Figure 5 shows that different

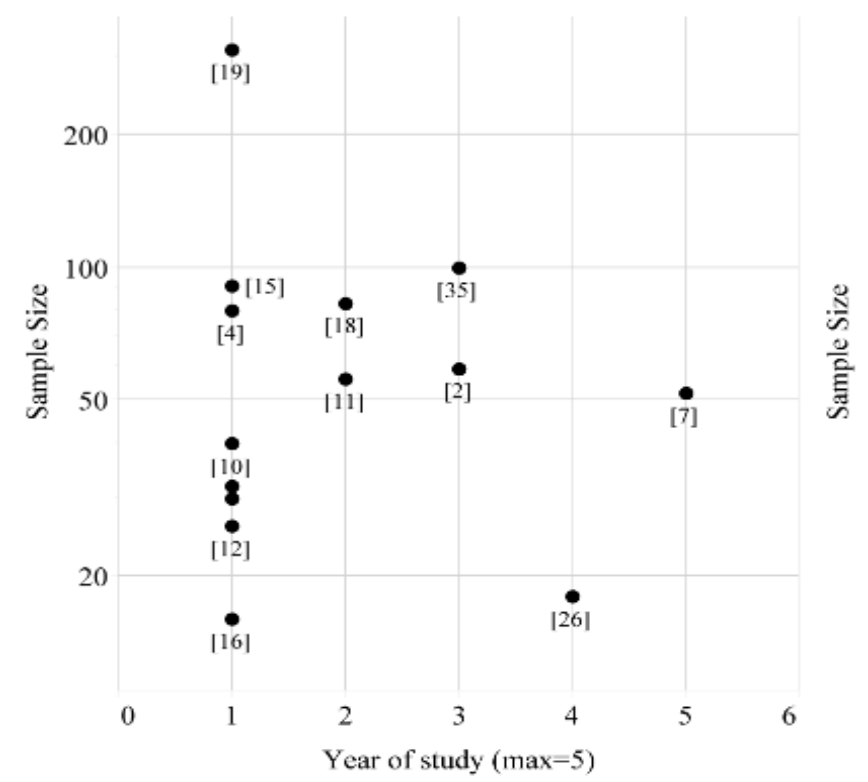

Figure 4. Sample size by year of study 
300

200

150

100

50

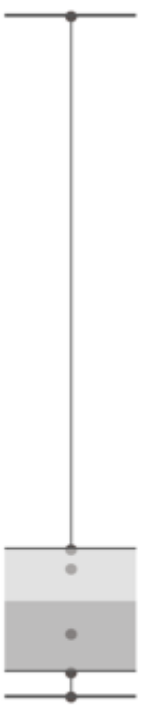

Experiment \& Quasi-experiment

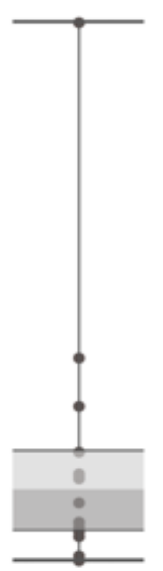

Pre/Pos

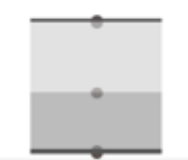

Cross-sectional

Figure 5. Sample size by research design

designs included differing sample size ranges: crosssectional samples where smaller (median=30) and experimental samples where larger $($ median $=70)$ compared with pre/post samples (median $=45$ ).

To explore this pattern, we observed the structure of research design quality and the main outcomes studied per design. Interestingly, almost $67 \%$ of the case presentations were placed either in the first or second quality quartiles (which can be interpreted as "optimal" and "good"), although this is true for just $36 \%$ of the texts that contain evaluations. No quasi-experimental design comes from a document deemed as "optimal" (considering that all the quasi-experimental studies included control groups, but only one was explicitly randomized, [18]). In parallel, $20 \%-33 \%$ of the literature was classified in the last quartile, irrespective of the research design followed. Secondly, we found that most quasi-experimental designs measured academic performance or less popular variables (spatial abilities in [19] and KPIs in [9]), compared with pre/post designs, that mainly focused on satisfaction measures, and crosssectional research, primarily interested in satisfaction and system performance measurement. This does not mean that these were the only exclusive possibilities, as shown in Table 10.

\section{Design Features}

On the following lines, we will describe the hardware and software listed in the literature. Most of the devices used by the literature were only Smartphones $(n=23$, $64 \%)$, or both Smartphone and PC/Tablets $(n=7,21 \%)$. The second most used device was the PC $(n=3,8 \%)$, followed by Tablets $(n=1,3 \%)$ and VR/AR mixes $(n=1$, $3 \%)$. Apparently, the found dominance of Smartphones in higher education is supported by the literature on STEM education-focused AR (Shirazi \& Behzadan, 2015) as opposed to reviews that include other education levels. On the other hand, earlier reviews state that teachers lean towards Junaio, ARMedia, and ARToolkit for designing their AR-based activities (Diao \& Shih, 2019; Sirakaya \& Sirakaya, 2018). It seems that LatinAmerican AR-based educational programs rather depend on Vuforia $(n=14,39 \%)$, Aumentary $(n=3,8 \%)$, Unity $(\mathrm{n}=3,8 \%)$, and ARToolkit-based $(\mathrm{n}=2,6 \%)$ applications. A small group $(\mathrm{n}=5,14 \%)$ even favored native applications, despite being a percentage fewer than the $43 \%$ reported by Ibáñez and Delgado-Kloos (2018); nonetheless, $63 \%(n=23)$ report or included some form of coding, including all applications based on Vuforia.

Diao and Shih (2019) stablish a difference between "general" and "specific purpose" AR software. Half of the applications reviewed by them where of "general" use (displaying text or graphics, or allowing 3D-object manipulation, for example), and the other half were of "specific use" (for object or architecture design, for example). On the other hand, drawing from literature about different education levels, Altinpulluk (2019) typified AR applications and found that most of them where 3D-Image based, Location-based, Video-based, games, or simulations and text based (from 17 overlapping types). In opposition to this literature, $71 \%$ $(n=25)$ of the applications in our review were of "general purpose" and mainly 3D-Image $(n=12)$, Text $(n=7)$, Simulation $(n=6)$, and Video-based $(n=3)$ software. Most of the general purpose software where largely 3D-Image

Table 10. Outcome variables and evaluation designs in the literature

\begin{tabular}{|c|c|c|c|}
\hline \multirow[b]{2}{*}{ Outcome variables measured } & \multicolumn{3}{|c|}{ Evaluation design } \\
\hline & Pre/Pos & Cross-sectional & $\begin{array}{c}\text { Experiment \& Quasi- } \\
\text { experiment }\end{array}$ \\
\hline Academic achievement & {$[7],[10]$} & & {$[15],[18]$} \\
\hline Satisfaction & $\begin{array}{c}{[3],[4],[5],[12],[13],} \\
{[24],[26],[35]}\end{array}$ & [25] & {$[31]$} \\
\hline Performance & & [33] & \\
\hline Academic achievement and satisfaction & {$[16],[21]$} & & {$[11],[30]$} \\
\hline Academic achievement and performance & {$[8],[32]$} & & \\
\hline Satisfaction and performance & [1] & [17] & \\
\hline Academic achievement, satisfaction, and performance & & {$[2]$} & \\
\hline Spatial skills, KPIs, and emotions & & {$[22]$} & [9], [19] \\
\hline
\end{tabular}




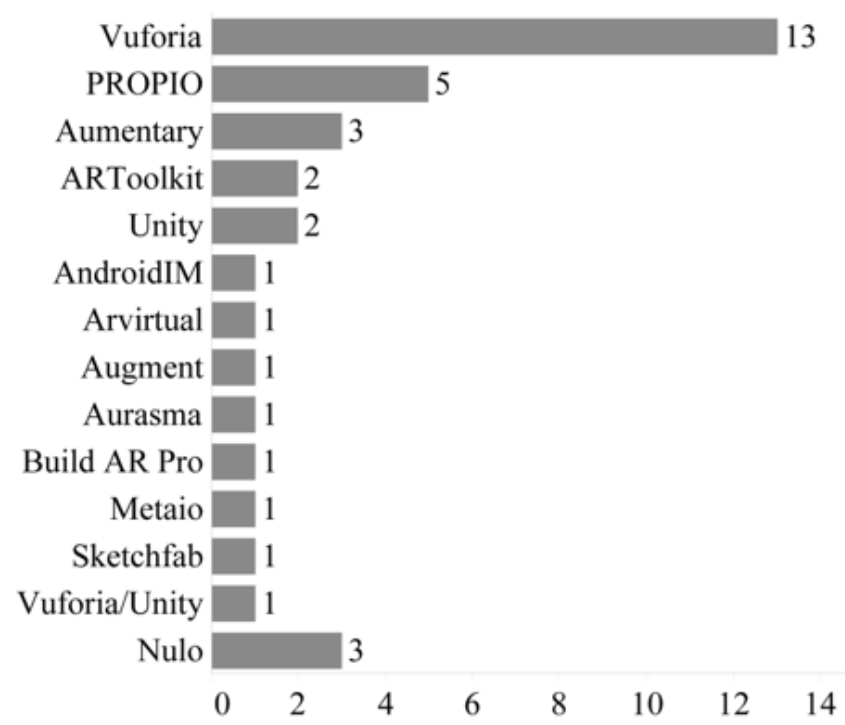

Figure 6. Distribution of documents according to software used

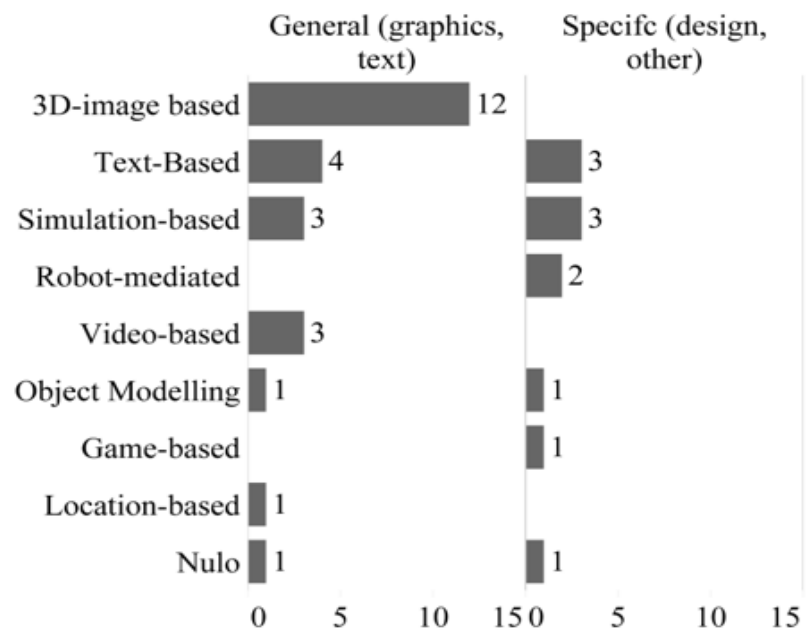

Figure 7. Number of documents by type of content and app type

$(n=12)$, Text $(n=4)$ and even Video-based $(n=3)$, while the other group was composed by mainly Text-based $(n=3)$, Simulation $(n=3)$ and Robot mediated $(n=2)$ software. Finally, no relevant differences were found between purpose and the use of native/non-native software. Figure 6 displays the distribution of documents according to software used while Figure 7 depicts the number of documents by type of content and app type.

The following paragraph describe additional AR software features in our engineering education literature. AR software based on marker or label recognition is predominant in the extant literature. We confirm this after finding 26 (72\%) marker-based, 3 (8\%) layer-based, and 2 (6\%) object-recognition software. In the same vein, drawing from de Belen et al. (2019), we delimited a three-step interaction continuum for AR technology. Our results show that a big part of our AR technology in our sample only allowed Perception $(n=16,44 \%)$, some endorsed Annotation $(n=6,17 \%)$ and the rest where based on interaction by direct Manipulation $(n=14,39 \%)$. In addition to this, following the findings of Montoya et al. (2016), we coded the presence of dynamic content $(n=15,42 \%)$, as opposed to static content. Though all observed application types had some dynamic content-focused examples, dynamic contents were only predominant among all Location and Video-based as well as most Simulation apps $(n=8,22 \%)$. Finally, out of the 20 documents reporting both considerable and secondary use of 3D-Objects or images, most were created by the teacher $(n=12)$, followed by those created by the class $(n=5)$ and those downloaded or already part of the employed software $(n=3)$.

\section{Pedagogy}

We coded the AR affordances and the main pedagogical perspectives linked with this technology. Saltan and Arslan (2016) suggested a seemingly useful categorization of three main AR pedagogical affordances. On the same line, AR in the reviewed literature afforded knowledge comprehension $(n=17$, $47 \%)$, concept development $(\mathrm{n}=14,39 \%)$, and learning retention $(n=5,14 \%)$. Secondly, perhaps the pedagogical perspectives that frame educational practices linked with AR are more difficult to define. Despite the lack of consensus, we identified two favored cognitivist frameworks, CTML $(n=13,36 \%)$ and Mobile Learning $(\mathrm{n}=9,25 \%)$, and three constructivist frameworks, Situated Learning $(\mathrm{n}=5,14 \%)$, Experiential Learning $(n=7,19 \%)$, and Collaborative Learning $(n=1,3 \%)$ (Sommerauer \& Müller, 2018). Examining the data, its easily seen that constructivist approaches favor AR concept development affordances in contrast with the other two. Interestingly, we also found a relationship between affordances and dynamic/static contents.

While most research engaged with engineering students, our literature populations pertained to a diversity of institutions: most of them to universities $(n=25,69 \%)$, some to technical schools (usually known as institutes, $n=8,22 \%)$, and a few to businesses $(n=2,6 \%)$. The latter were more prone to engage with a cognitivist framework, but half of the AR-related practices in institutes were constructivist. Besides, we analyzed teaching and academic evaluation practices related with $\mathrm{AR}$, finding out that $78 \%$ were task-based and $69 \%$ $(n=25)$ were problem-solving-focused activities (Diao \& Shih, 2019; Wu et al., 2013). Following our analysis, we correspondingly saw the importance of technical schools for experimenting with more collaborative approaches to teaching (whether role or location-based) and evaluation activities (e. g., group or pair projects, peerbased work, etc.): most of the synchronic task-based activities ([30], [35], [34]) and the only group projectbased course ([21]) were done in these institutions. Even if this trend contrasts with the project-based pedagogy prevalent in other AR education contexts (Diao \& Shih, 2019), the relationship between constructivism and 
Centro ed..

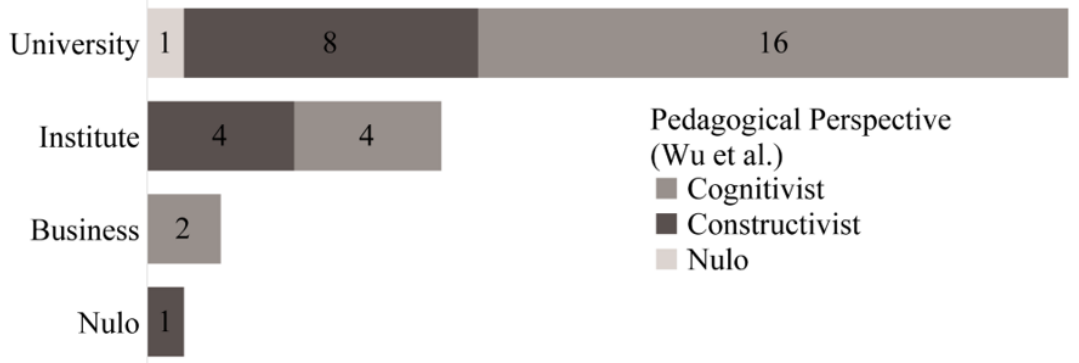

Figure 8 . Number of documents by pedagogical perspective and post-secodary education institution
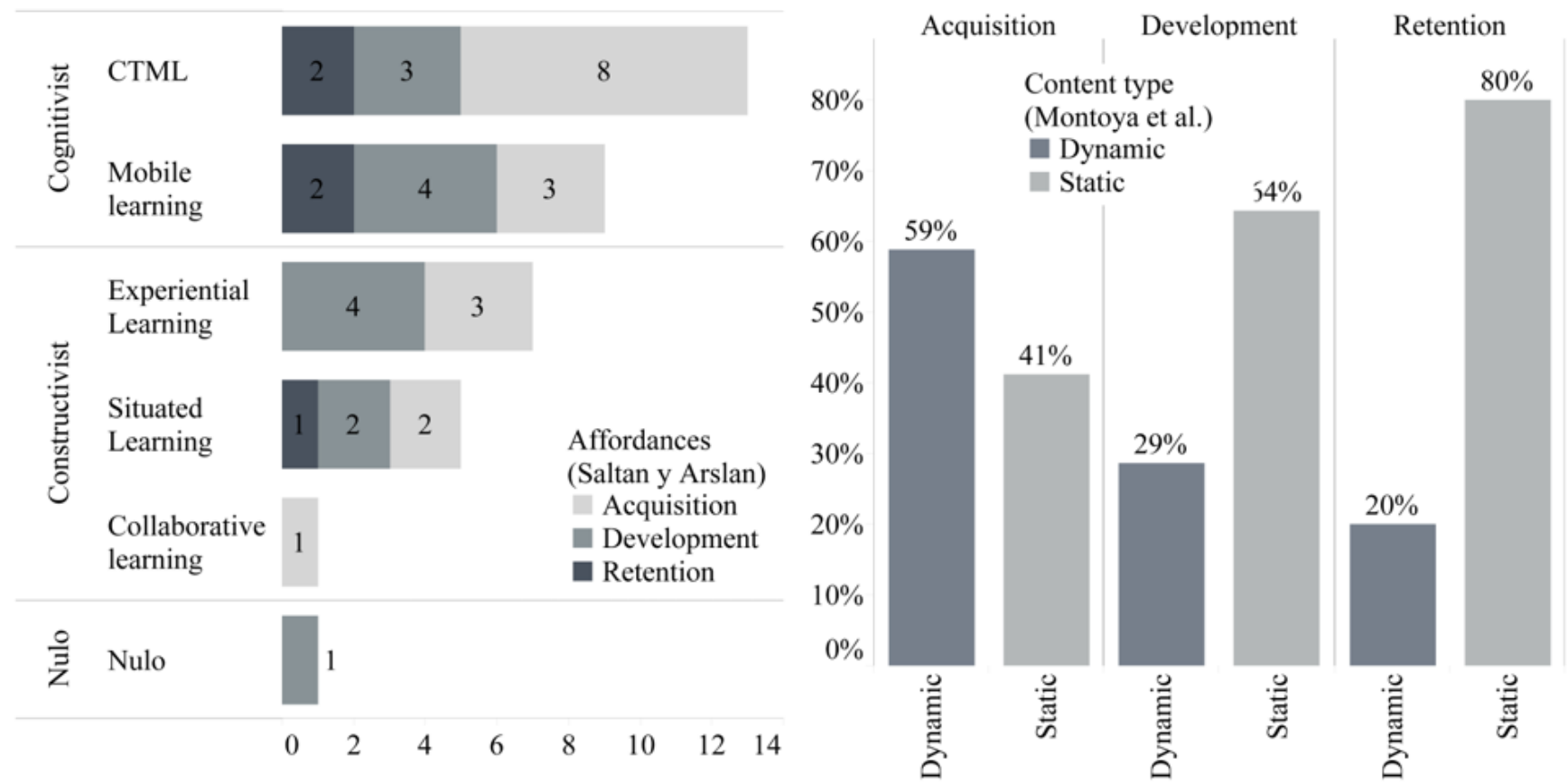

Figure 9. Number (left) and percentage (right) of documents according to their educational affordances by pedagogical perspective and content type

collaborative learning became apparent when we saw that the only remote-based collaborative course found ([21]) was supported by an institute (de Belen et al., 2019). Figure 8 shows the number of documents by pedagogical perspective and post-secodary education institution.

Another way to look at this is to understand the kind of pedagogical experiences that students undergo when using AR. Following Chubukova and Ponomarenko (2018), these can be: modeling situations ( $n=14,39 \%)$, acquiring skills $(n=12,33 \%)$, learning with textbooks or manuals $(n=4,11 \%)$, game-like experiences $(n=3,8 \%)$, and 3D object modelling $(n=2,6 \%)$. We saw that skill training and game-alike experiences are the only ones that partly support knowledge retention, however content acquisition is helped by all experiences except for game-alike, and concept development is only entirely absent of textbook/manual-based experiences. On the other hand, it is interesting to note that dynamic contents are a minority in all experiences, except for modelling. Figure 9 depicts the number and percentage of documents according to their educational affordances by pedagogical perspective and content type.

What are the main advantages and disadvantages of the use of AR in engineering education? In our review, most authors $(n=15)$ agreed that AR motivated students $(n=15)$, followed by those who valued an increase in academic achievement $(n=11)$, the ease of use $(n=9)$, innovativeness $(n=6)$ and collaboration $(n=2)$. Interestingly, more authors with ideas closer to experiential and situated learning report motivation benefits; whereas, among those reporting increases in academic achievement, the mobile learning framework is more common. In spite of a common consensus of AR being beneficial for academic achievement among other advantages (Akcayir \& Akcayir, 2017; Bacca et al., 2014; Singh et al., 2019), a recent meta-analysis point towards the more nuanced conclusion that AR actually helps student engagements and abstract concept understanding (Garzón et al., 2019; Liono et al., 2021).

To conclude, virtually all authors mentioned an advantage, but less than half $(n=16)$ mentioned 
disadvantages, namely heterogeneous benefits for different types of users $(n=6)$, demanding technical requirements $(n=6)$, accessibility issues related with skill gaps (especially among teachers and older professionals, $n=5)$, the complexity of the setups used $(n=2)$ and pedagogical insufficiencies $(n=1)$. Both the lack of limitations and the complexity and technical problems have been found before in the AR literature (Akcayir \& Akcayir, 2017; Bacca et al., 2014).

\section{DISCUSSION}

AR is a nowadays considered a mainstream tool for engineering education in Latin America (Hidrogo et al., 2020). Although this technology enhances important research, social and work-related skills in higher education (Klimova et al., 2018), questions about humanbased design, display technology, pedagogy and collaboration remain open (Billinghurst, 2021). In this work we reviewed conference papers and scientific articles published by Latin-American authors, focusing on AR uses in engineering education. Even though others reviewed experiences from different educational levels and disciplines, we tried to tackle many of the still open themes while only focusing on higher education.

One of the reasons to do this was to rethink the role of innovation to address the current knowledge gaps in the world. We found an increasing number of quality indexed conferences and a stagnant number of articles written by mainly Mexican, Colombian, and Brazilian authors. Even though most of the literature presented medium quality evaluations, different research designs seem to relate with corresponding sample sizes, variables measured and data collection techniques. At the same time, Latin-American engineering educators prefer conventional open-source AR software and Smartphone devices, incorporating some basic coding and 3D object modelling; however, we reported a big interest for manipulation and annotation based applications, as well as important object recognition software applications. Pedagogically, most university AR-related engineering programs and activities engage with cognitivist frameworks, but institutes seem to be embracing the emergence of constructivist and collaborative innovations. In general, authors highlight motivation academic achievement advantages, but overlook the disadvantages; when acknowledged, they emphasize accessibility and technical issues.

These findings integrate with the literature in two important ways. First, we can support the view that this literature leaves aside a needed focus on accessibility and longitudinal approaches (Bacca et al., 2014). Nonetheless, Latin-American authors, especially those affiliated with institutes, tackle, at least partly, collaboration, interaction issues and other largely overlooked UX design issues, as well as vocational learning, in a very intermingled way (Bacca et al., 2018;
Ibáñez \& Delgado-Kloos, 2018; Phon et al., 2014; Shirazi \& Behzadan, 2015). These innovative authors seem likely interested in the motivational benefits of game and simulation-based learning (Ayer et al., 2016). Yet, contradicting earlier reviews, this trend is far from the mainstream. Our review also revealed a delay in evidence-based pedagogical practices, especially within universities: few authors seem interested in randomized controlled trials or mixed methods, and task-based evaluation practices within cognitivist pedagogies are still preferred over newer approaches.

We further believe to have shown the value of reviewing conference papers along with scientific articles. This helped us to learn about the importance of contextual factors before making assumptions about the advancement of Industry 4.0 technologies through ARbased engineering education (Hernandez-de-Menendez et al., 2020). We think that Latin-American university educators, which are the greatest part of our sample, prefer to report conventional AR uses under cognitivist approaches, in contrast with other technologies and pedagogies, given the cost of Smartphones for their students, the limitations of their university budgets, the accessibility of open-source 3D object modeling and AR software, and the greater simplicity of conference formats in contrast with the demanding formats of international journals.

The limitations of the following review include proceeding without a pairwise quality assessment, applying a largely experimental quality assessment tool (and including some low-quality documents, due to the nature of scoping reviews), unavertedly or intentionally over-simplifying non-exclusive categories of certain variables, and having worked against time with an extensive number of research questions and variables. Future reviews should a) attend to relevant or influential pedagogical and/or technological innovations in engineering education in different global regions, b) discover the barriers for the adoption of such innovations by more precise literature review questions and informative methods (ranging from meta-analyses to multivocal reviews), and c) develop recommendations to better manage the knowledge production in different higher education institutions. Finally, we confirm the lack of longitudinal studies, the small quantity of correlational and experimental research, and very few direct references to qualitative methodologies, which justifies future additional research.

\section{CONCLUSIONS}

This scoping review shows that the accumulating Latin-American literature regarding the use of AR in engineering education is mostly pedagogically and technologically conservative, and that the research designs behind this literature are diverse but still limited. Nevertheless, we believe to have found a 
positive and emerging trend among institute-based engineering education. Moreover, using a literaturebased categorization, we found a diversity of application types and contents, contradicting the international trends in certain aspects, and even finding various direct mentions of software coding in all the literature. We also find that most advances are reported as mostly Scopusindexed conference papers, which is the only literature type in expansion.

We believe that these results inform the management of STEM education policies in the region. Knowledge gaps around the world, including those in research quality, are relevant to the diffusion of innovations in engineering education. Universities and teachers might consider accessibility and performance issues when trying out AR-based courses, but also should experience more with other pedagogies and forms of evaluation. Finally, future literature reviews might consider our solutions to the lack of representation of developing regions, as well as the differences between international patterns and locally-based phenomena.

Author contributions: All authors have sufficiently contributed to the study, and agreed with the results and conclusions.

Funding: No funding source is reported for this study.

Acknowledgements: The authors thank their families and authorities from USIL for their constant support and encouragement. We specially thank Diego Paucar from ESCAPE, who guided us in the design, analysis, write-up and translation stages of this research. For all his effort, we are in great debt with him.

Declaration of interest: No conflict of interest is declared by authors.

\section{REFERENCES}

Adams, R. J., Smart, P., \& Huff, A. S. (2017). Shades of grey: Guidelines for working with the grey literature in systematic reviews for management and organizational studies. International Journal of Management Reviews, 19(4), 432-454. https://doi.org/10.1111/ijmr.12102

Akcayir, M., \& Akcayir, G. (2017). Advantages and challenges associated with augmented reality for education: A systematic review of the literature. Educational Research Review, 20, 1-11. https://doi.org/10.1016/j.edurev.2016.11.002

Aljawarneh, S. A. (2020). Reviewing and exploring innovative ubiquitous learning tools in higher education. Journal of Computing in Higher Education, 32(1), 57-73. https://doi.org/10.1007/s12528-01909207-0

Altinpulluk, H. (2019). Determining the trends of using augmented reality in education between 2006-2016. Education and Information Technologies, 24(2), 10891114. https:/ / doi.org/10.1007/s10639-018-9806-3

Alyahyan, E., \& Dustegor, D. (2020). Predicting academic success in higher education: Literature review and best practices. International Journal of
Educational Technology in Higher Education, 17(1), 3. https:/ / doi.org/10.1186/s41239-020-0177-7

Ayer, S. K., Messner, J. I., \& Anumba, C. J. (2016). Augmented reality gaming in sustainable design education. Journal of Architectural Engineering, 22(1), 04015012. https://doi.org/10.1061/(ASCE)AE. 1943-5568.0000195

Bacca, J., Baldiris, S., Fabregat, R., \& Kinshuk. (2018). Insights into the factors influencing student motivation in augmented reality learning experiences in vocational education and training. Frontiers in Psychology, 9. https:/ / doi.org/10.3389/ fpsyg.2018.01486

Bacca, J., Baldiris, S., Fabregat, R., Graf, S., \& Kinshuk. (2014). Augmented reality trends in education: A systematic review of research and applications. Educational Technology \& Society, 17(4), 133-149. http://www.jstor.org/stable/jeductechsoci.17.4.1 33

Barroso Osuna, J., Gutiérrez-Castillo, J. J., LlorenteCejudo, M. del C., \& Valencia Ortiz, R. (2019). Difficulties in the incorporation of augmented reality in university education: Visions from the experts. Journal of New Approaches in Educational Research, 8(2), 126. https://doi.org/10.7821/ naer.2019.7.409

Bellido García, R. S., \& Paucar Villacorta, D. M. (2021). The use of augmented reality (AR) in engineering education in Latin America: A scoping review. https://osf.io/w7hk4

Billinghurst, M. (2021). Grand challenges for augmented reality. Frontiers in Virtual Reality, 2. https:/ / doi.org/10.3389/frvir.2021.578080

Chubukova, O. S., \& Ponomarenko, I. (2018). Innovatsiyni tekhnolohiyi dopovnenoyi real'nosti dlya vykladannya dystsyplin $u$ vyshchykh navchal'nykh zakladakh Ukrayiny [Innovative technologies of augmented reality for teaching disciplines in higher educational institutions of Ukraine]. Problemy innovatsiyno-investytsiynoho rozvytku [Problems of Innovation and Investment Development], 16. https://er.knutd.edu.ua/handle /123456789/11227

de Belen, R. A. J., Nguyen, H., Filonik, D., Favero, D. D., Bednarz, T., de Belen, R. A. J., Nguyen, H., Filonik, D., Favero, D. D., \& Bednarz, T. (2019). A systematic review of the current state of collaborative mixed reality technologies: 2013-2018. AIMS Electronics and Electrical Engineering, 3(2), 181-223. https:/ / doi.org/10.3934/ElectrEng.2019.2.181

Diao, P.-H., \& Shih, N.-J. (2019). Trends and research issues of augmented reality studies in architectural and civil engineering education-A review of academic journal publications. Applied Sciences, 9(9), 1840. https:// doi.org/10.3390/app9091840 
Drew, D. E. (2020). STEM education, economic productivity, and social justice. In D. E. Drew (Ed.), Oxford Research Encyclopedia of Education. Oxford University Press. https://doi.org/10.1093/ acrefore/9780190264093.013.738

Garousi, V., Felderer, M., \& Mäntylä, M. V. (2019). Guidelines for including grey literature and conducting multivocal literature reviews in software engineering. Information and Software Technology, 106, 101-121. https://doi.org/10.1016/ j.infsof.2018.09.006

Garzón, J., Pavón, J., \& Baldiris, S. (2019). Systematic review and meta-analysis of augmented reality in educational settings. Virtual Reality, 23(4), 447-459. https:/ / doi.org/10.1007/s10055-019-00379-9

Gómez-Tone, H. C., Martin-Gutierrez, J., Valencia Anci, L., \& Mora Luis, C. E. (2020). International comparative pilot study of spatial skill development in engineering students through autonomous augmented reality-based training. Symmetry, 12(9), 1401. https://doi.org/10.3390/ sym12091401

Greenan, K. A. (2021). The influence of virtual education on classroom culture. Frontiers in Communication, 6. https://doi.org/10.3389/fcomm.2021.641214

Hartling, L., Featherstone, R., Nuspl, M., Shave, K., Dryden, D. M., \& Vandermeer, B. (2017). Grey literature in systematic reviews: A cross-sectional study of the contribution of non-English reports, unpublished studies and dissertations to the results of meta-analyses in child-relevant reviews. BMC Medical Research Methodology, 17(1), 64. https: / / doi.org/10.1186/s12874-017-0347-z

Hernandez-de-Menendez, M., Escobar Díaz, C. A., \& Morales-Menendez, R. (2020). Engineering education for smart 4.0 technology: A review. International Journal on Interactive Design and Manufacturing, 14(3), 789-803. https://doi.org/ 10.1007 / s12008-020-00672-x

Hidrogo, I., Zambrano, D., Hernandez-de-Menendez, M., \& Morales-Menendez, R. (2020). Mostla for engineering education: Part 2 emerging technologies. International Journal on Interactive Design and Manufacturing, 14(4), 1461-1473. https:/ / doi.org/10.1007/s12008-020-00729-x

Iatsyshyn, A., Kovach, V., Romanenko, Y., Deinega, I., Iatsyshyn, A., Popov, O., Kutsan, Y., Artemchuk, V., Burov, O., \& Lytvynova, S. (2020). Application of augmented reality technologies for preparation of specialists of new technological era. Proceedings of the 2nd International Workshop on Augmented Reality in Education, 181-200. http:/ / ds.knu.edu.ua /jspui/handle/123456789/2184

Ibáñez, M.-B., \& Delgado-Kloos, C. (2018). Augmented reality for STEM learning: A systematic review.
Computers $\mathcal{E} \quad$ Education, 123, 109-123. https://doi.org/10.1016/j.compedu.2018.05.002

Isaksson, O., Eckert, C., Panarotto, M., \& Malmqvist, J. (2020). You need to focus to validate. Proceedings of the Design Society: DESIGN Conference, 1, 31-40. https://doi.org/10.1017/dsd.2020.116

Kitchenham, B., \& Brereton, P. (2013). A systematic review of systematic review process research in software engineering. Information and Software Technology, 55(12), 2049-2075. https://doi.org/ 10.1016/j.infsof.2013.07.010

Klimova, A., Bilyatdinova, A., \& Karsakov, A. (2018). Existing teaching practices in augmented reality. Procedia Computer Science, 136, 5-15. https://doi.org/10.1016/j.procs.2018.08.232

Liono, R. A., Amanda, N., Pratiwi, A., \& Gunawan, A. A. S. (2021). A systematic literature review: Learning with visual by the help of augmented reality helps students learn better. Procedia Computer Science, 179, 144-152. https://doi.org/10.1016/j.procs.2020.12. 019

Liu, S., Fang, Z., Shi, H., \& Guo, B. (2016). Theory of Science and Technology Transfer and Applications. CRC Press. https://doi.org/10.1201/97814200 87420

Mårtensson, P., Fors, U., Fröberg, E., Zander, U., \& Nilsson, G. H. (2019). Quality of research practice An interdisciplinary face validity evaluation of a quality model. PLOS ONE, 14(2), e0211636. https:/ / doi.org/10.1371/journal.pone.0211636

Mkrttchian, V., Krevskiy, I., Bershadsky, A., Glotova, T., Gamidullaeva, L., \& Vasin, S. (2019). Web-based learning and development of university's electronic informational educational environment. International Journal of Web-Based Learning and Teaching Technologies, 14(1), 32-53. https:/ / doi.org/ 10.4018/IJWLTT.2019010103

Montoya, M. H., Díaz, C. A., \& Moreno, G. A. (2016). Evaluating the effect on user perception and performance of static and dynamic contents deployed in augmented reality based learning application. EURASIA Journal of Mathematics, Science and Technology Education, 13(2). https:/ / doi.org/10.12973/eurasia.2017.00617a

Munn, Z., Peters, M. D. J., Stern, C., Tufanaru, C., McArthur, A., \& Aromataris, E. (2018). Systematic review or scoping review? Guidance for authors when choosing between a systematic or scoping review approach. BMC Medical Research Methodology, 18(1), 143. https://doi.org/10.1186/ s12874-018-0611-x

Nesenbergs, K., Abolins, V., Ormanis, J., \& Mednis, A. (2021). Use of augmented and virtual reality in remote higher education: A systematic umbrella 
review. Education Sciences, 11(1), https:// doi.org/10.3390/educsci11010008

Peters, M. D. J., Marnie, C., Tricco, A. C., Pollock, D., Munn, Z., Alexander, L., McInerney, P., Godfrey, C. M., \& Khalil, H. (2020). Updated methodological guidance for the conduct of scoping reviews. JBI Evidence Synthesis, 18(10), 2119-2126. https:/ / doi.org/10.11124/JBIES-20-00167

Petersen, K. (2020). Guidelines for case survey research in software engineering. In M. Felderer \& G. H. Travassos (Eds.), Contemporary empirical methods in software engineering (pp. 63-92). Springer International Publishing. https:/ / doi.org/10.1007/ 978-3-030-32489-6_3

Phon, D. N. E., Ali, M. B., \& Halim, N. D. A. (2014) Collaborative augmented reality in education: A review. 2014 International Conference on Teaching and Learning in Computing and Engineering, 78-83. https:// doi.org/10.1109/LaTiCE.2014.23

Saltan, F., \& Arslan, O. (2016). The use of augmented reality in formal education: A scoping review. Eurasia Journal of Mathematics, Science and Technology Education, 13(2), 503-520. https:// doi.org/10.12973/eurasia.2017.00628a

Schön, E.-M., Thomaschewski, J., \& Escalona, M. J. (2017). Agile requirements engineering: A systematic literature review. Computer Standards $\mathcal{E}$ Interfaces, 49, 79-91. https://doi.org/10.1016/ j.csi.2016.08.011

Shirazi, A., \& Behzadan, A. H. (2015). Content delivery using augmented reality to enhance students performance in a building design and assembly project. Advances in Engineering Education, 4(3). https:/ / eric.ed.gov/?id=EJ1076141

Singh, G., Mantri, A., Sharma, O., Dutta, R., \& Kaur, R. (2019). Evaluating the impact of the augmented reality learning environment on electronics laboratory skills of engineering students. Computer Applications in Engineering Education, 27(6), 13611375. https:/ / doi.org/10.1002/cae.22156

Sirakaya, M., \& Sirakaya, D. A. (2018). Trends in educational augmented reality studies: A systematic review. Malaysian Online Journal of Educational Technology, 6(2), 60-74. https:/ / doi.org/ 10.17220 / mojet.2018.02.005
8. Sirakaya, M., \& Sirakaya, D. A. (2020). Augmented reality in STEM education: A systematic review. Interactive Learning Environments, 0(0), 1-14. https:/ / doi.org/10.1080/10494820.2020.1722713

Sommerauer, P., \& Müller, O. (2018). Augmented reality for teaching and learning - A literature review on theoretical and empirical foundations. Research Papers, 31. https:/ / aisel.aisnet.org/ecis2018_rp/31

Tricco, A. C., Lillie, E., Zarin, W., O'Brien, K. K., Colquhoun, H., Levac, D., Moher, D., Peters, M. D. J., Horsley, T., Weeks, L., Hempel, S., Akl, E. A., Chang, C., McGowan, J., Stewart, L., Hartling, L., Aldcroft, A., Wilson, M. G., Garritty, C., ... Straus, S. E. (2018). PRISMA Extension for Scoping Reviews (PRISMA-ScR): Checklist and Explanation. Annals of Internal Medicine, 169(7), 467473. https:/ / doi.org/10.7326/M18-0850

UNESCO. (2021). UNESCO science report: The race against time for smarter development. UNESCO. https:/ / en.unesco.org/unescosciencereport

Wang, M., Callaghan, V., Bernhardt, J., White, K., \& Peña-Rios, A. (2018). Augmented reality in education and training: Pedagogical approaches and illustrative case studies. Journal of Ambient Intelligence and Humanized Computing, 9(5), 13911402. https:/ / doi.org/10.1007/s12652-017-0547-8

Wang, P., Zhang, S., Billinghurst, M., Bai, X., He, W., Wang, S., Sun, M., \& Zhang, X. (2020). A comprehensive survey of AR/MR-based co-design in manufacturing. Engineering with Computers, 36(4), 1715-1738. https:// doi.org/10.1007/s00366019-00792-3

Wu, H.-K., Lee, S. W.-Y., Chang, H.-Y., \& Liang, J.-C. (2013). Current status, opportunities and challenges of augmented reality in education. Computers $\mathcal{E}$ Education, 62, 41-49. https://doi.org/10.1016/ j.compedu.2012.10.024

Zawacki-Richter, O., Marín, V. I., Bond, M., \& Gouverneur, F. (2019). Systematic review of research on artificial intelligence applications in higher education - where are the educators? International Journal of Educational Technology in Higher Education, 16(1), 39. https://doi.org/ 10.1186/s41239-019-0171-0

\section{https://www.ejmste.com}

